\author{
ANNALES \\ POLONICI MATHEMATICI \\ XLII (1983)
}

\title{
Conditionally positive definite functions on linear spacę
}

\author{
by W. MLaK (Kraków)
}

Dedicated to the memory of Jacek Szarski

\begin{abstract}
The first part of the paper deals with general properties and related dilations of conditionally positive definite functions $b_{x, y}(f, g)$ on a linear space. Next, the Levy-Khintehine formula is proved for $b_{x-y}(f, g)$, where $x, y$ vary over a separable real Hilbert space. We deduce therefore the Yaglom formula for related helix and describe then the corresponding shift group for Poisson type functions.
\end{abstract}

The present paper has its origin in the desire to present some new dilation type phenomena related to conditional positive definiteness. The point is that we discuss functions $b_{x, y}(f, g)$ with $x, y$ varying over a some set $X$ and $f, g$ are in a linear space $\mathscr{E} ; b_{x, y}(f, g)$ is bilinear in $f, g$ and we can assume that for each $f$ the function $(x, y) \rightarrow b_{x, v}(f, f)$ is conditionally positive definite (weak conditional positive definiteness). The stronger property is defined, namely the strong version, which reads as follows: for any $n, x_{1}, \ldots, x_{n} \in X$ and $f_{1}, \ldots, f_{n} \in \mathscr{E}$ the inequality $\sum_{j, k} b_{x_{j}, x_{k}}\left(f_{j}, f_{k}\right) \geqslant 0$ holds true provided that $\sum_{i \mid 1}^{n} f_{i}=0$. The "strong version" makes it possible to apply the technique of dilation theory (we refer here to [19]-[22] and [33]) and just get theorems which extend in some sense classical results, i.e. that ones when $f, g$ run over the field of complex scalars and $b_{x, y}(f, g)=b_{x, y} f \cdot \bar{g}$, where $b_{x, y}$ is a scalar function.

In order to make this paper reasonably self-contained, sketches of those results of general dilation theory which we need are given with suitable comments. On the other hand we try to give in a rather complete way some technical arguments we need, especially those concerning infinitely divisible random variables. These arguments are "almost" surely routine, but the often incomplete (if not false) way of their presentation in the literature plus the method of "succesive shifting of references" makes the life of reader not easy - this is the reason we describe in some detail the so-called "well-known" ("trivial") facts. We need them both 
with their proofs, when applying the general theory of initial section to functions $b_{x-y}(f, g)(f, g \in \mathscr{E})$ with $x, y$ varying over a real separable Hilbert space $X$. The final part of the paper deals with some continuity properties in $f, g$ when $\mathscr{E}$ is a metric space. We restrict ourselves to such $\mathscr{E}$ for the sake of simplicity as well to get some nice and compact operator versions of Levy-Khintchine formula in case when $X$ is finite dimensional.

1. Notation and definitions. In all what follows $\mathscr{E}$ stands for a complex linear space. The function $l: \mathscr{E} \times \mathscr{E} \rightarrow C$ is called bilinear form on $\mathscr{E}$ if

$$
l(\alpha f+\beta g, h)=\alpha l(f, h)+\beta l(g, h), \quad l(f, \alpha g+\beta h)=\bar{a} l(f, g)+\bar{\beta} l(f, h)
$$

for $a, \beta \in C$ and $f, g, h \in \mathscr{E}$.

The bilinear form $l$ can be interpreted in terms of linear operators, namely $l$ is bilinear if and only if there is the unique linear operator $A_{l}$, which maps $\mathscr{E}$ into the set $\mathscr{E}^{*}$ of all antilinear functionals on $\mathscr{E}$, such that $l(f, g)=\left(A_{l} f\right)(g)$ for all $f, g \in \mathscr{E}$.

Let $X$ be the set with typical elements $x, y, z, \ldots$, etc. Suppose once for all through the whole paper that if for every $x, y \in X$ we are given the bilinear function $l_{x, y}(f, g)$ on $\mathscr{E}$, then we write formally $l=\left\{l_{x, y}(f, g)\right\}$.

Definition 1.0. We say that the function $l=\left\{l_{x, y}(f, g)\right\}$ is weakly positive definite if for every $f \in \mathscr{E}$ and for every $n=1,2, \ldots$ and arbitrary $a_{1}, \ldots, \alpha_{n} \in C$ the inequality $\sum_{j, k \mid 1}^{n} l_{x_{j}, x_{k}}(f, f) \alpha_{j} \bar{a}_{k} \geqslant 0$ holds true for any $x_{1}, \ldots$
..,$x_{n} \in X$.

Definimion 1.1. We say that the function $l=\left\{l_{x, y}(f, g)\right\}$ is positive definite (and write then $l>>0$ or $l_{x, y}(\cdot, \cdot)>>0$ ) if for every $n=1,2, \ldots$, for any $f_{1}, \ldots, f_{n} \in \mathscr{E}$ and arbitrary $x_{1}, \ldots, x_{n} \in X$ the inequality $\sum_{j, k \mid 1}^{n} l_{x_{j}, x_{k}}\left(f_{j}, f_{k}\right) \geqslant 0$ holds true.

If $l_{x, y}(\cdot, \cdot)>>0$, then $l$ is hermitian symmetric, i.e.

$$
l_{x, y}(f, g)=\overline{l_{y, x}(g, f)} \quad \text { for } x, y \in X ; f, g \in \mathscr{E} \text {. }
$$

Let $K$ be a complex Hilbert space and $Z_{s} \subset K(s \in S)$ a family of subsets of $K$. We write $K=\bigvee_{s \in S} Z_{s}$ if the linear span of the union $\bigcup_{s \in S} Z_{s}$ is dense in $K$.

2. Canonical representation. To every positive definite function $l=\left\{l_{x, y}(f, g)\right\}$ there corresponds in the canonical fashion a Hilbert space $K$ and a family of linear maps from $\mathscr{E}$ into $K$, a family indexed by $x$-es from $X$. The corresponding theorem will be referred as the KMKA Lemma, because its essential part is due to Kolmogorov [11], Moore-Aronszajn - see [1] and Krein [14]; the operator version of the KMKA Lemma is explicitly formulated in Koranyi-Sz.-Nagy [12] and Kunze [15] for operator func- 
tions in Hilbert spaces and by Pedrick [28] in a more general setting. Masani was who in [20] formulated the KMKA Lemma under some general circumstances (showing its links with results of Kolmogorov and Aronszajn) and showed explicitly its connection with the dilation theory. On the other hand, the "scalar" version of the KMKA Lemma is a "foiklore" in probability theory - see Proposition 2.0 below.

KMKA LEMma. Suppose that we are given the function $l=\left\{l_{x, y}(f, g)\right\}$ such that $l_{x, y}(\cdot, \cdot)>>0$. Then there is a complex Hilbert space $K$ with inner product $(\cdot, \cdot)_{K}$ and a family of linear maps $Y(x)(x \in X)$ defined on $\mathscr{E}$ with values in $K$ such that

$$
l_{x, y}(f, g)=(Y(x) f, Y(y) g)_{K} \quad \text { for } x, y \in X ; f, g \in \mathscr{E} .
$$

Moreover, $K$ can be chosen minimal, i.e. such that $K=\underset{x \in X}{\bigvee} Y(x) \mathscr{E}$. If $K$ is minimal, then it is unique up to unitary equivalence in the following sense: if $K^{\prime}$ is some other complex Hilbert space, $\bar{Y}^{\prime}(x)$ are linear maps from $\mathscr{E}$ in $K^{\prime}, K^{\prime}=\bigvee_{x \in X} \Gamma^{\prime}(x) \mathscr{E}$ and $l_{x, y}(f, g)=\left(\bar{Y}^{\prime}(x) f, \bar{Y}^{\prime}(y) g\right)_{K^{\prime}}$ for $x, y \in X$; $f, g \in \mathscr{E}$, then there is a unitary map $U: K \rightarrow K^{\prime}$ such that $U Y(x)=Y^{\prime}(x)$ for all $x \in X$.

Proof. Let $S$ be the complex linear space of $\mathscr{E}$-valued functions $\hat{f}(x)$ defined on $X$ with pointwise linear operations and such that $\hat{f}(x) \neq 0$ for at most a finite number of $x$-es. Since $l$ is positive definite, the bilinear form

$$
\langle\hat{f}, \hat{g}\rangle=\sum_{x, y} l_{x, y}(\hat{f}(x), \hat{g}(y))
$$

is a semi-immer product on $S$. It follows now from the Schwarz inequality, that the set $S_{0}=\{f \in S:\langle\hat{f}, \hat{f}\rangle=0\}$ is a linear subspace of $S$. Define now $K=$ completion of the quotient space $S / S_{0}$ to the Hilbert space. Then $Y(x) f=$ the equivalence class corresponding to $\hat{f}_{x}(y)=\delta_{x}(y) f$ does the trick, because

$$
(Y(x) f, Y(y) g)_{K}=\left(\hat{f}_{x}(\cdot), \hat{g}_{y}(\cdot)\right)_{K}=\sum_{u, v \in X} l_{u, v}\left(\delta_{x}(u) f, \delta_{y}(v) g\right)=l_{x, y}(f, g)
$$

and the functions $\delta_{x}(\cdot) f(x \in X, f \in \mathscr{E})$ span algebraically $S$.

In order to complete the proof we assume that $K^{\prime}$ and $Y^{\prime}(x)$ satisfy all we said. Then, for any $n, x_{1}, \ldots, x_{n}, y_{1}, \ldots, y_{n} \in X, f_{1}, \ldots, f_{n}, g_{1}, \ldots$ $\ldots, g_{n} \in \mathscr{E}$ we have

$$
\begin{aligned}
\left(\sum_{j} Y\left(x_{j}\right) f_{j}, \sum_{k} Y\left(y_{k}\right) g_{k}\right)_{K} & =\sum_{j, k} l_{x_{j}, x_{k}}\left(f_{j}, g_{k}\right) \\
& =\left(\sum_{j} Y^{\prime}\left(x_{j}\right) f_{j}, \sum_{k} Y^{\prime}\left(y_{k}\right) g_{k}\right)_{K^{\prime}}
\end{aligned}
$$


which, since $K$ and $K^{\prime}$ are minimal, proves that there is the unique unitary map $U: K \rightarrow K^{\prime}$ which sends $Y(x) f$ onto $Y^{\prime}(x) f$ for $x \in X, f \in \mathscr{E}$.

The equality $K=\bigvee_{x \in X} Y(x) \mathscr{E}$ is called the minimality condition. The final statement of KMKA Lemma may be stated as follows: the minimality condition determines $K$ and $Y(\cdot)$ satisfying (2.0) in a unique way up to unitary equivalence. Formula $(2.0)$ is called therefore the canonical representation of $l$ if $K$ is minimal. If $l_{x, y}(f, g)=(B(x, y) f, g)$, where $\mathscr{E}$ is a Hilbert space and $B(x, y)$ are linear bounded operators in $\mathscr{E}$, then $(2.0)$ implies that $Y(x)$ are linear bounded operators and in fact (2.0) reduces to the formula

$$
B(x, y)=Y(y)^{*} Y(x) \quad(x, y \in X)
$$

which is called the canonical factorization of $B(\cdot, \cdot)$.

The KMKA Lemma can be expressed and proved in probabilistic terms, by using the celebrated Kolmogorov's theorem on consistent families of measures and the fact, that every positive definite finite matrix is a covariance matrix of a complex Gaussian variable. However, the use of the Kolmogorov theorem in its full generality in a such approach is a certain kind of abuse, simply because it's weaker version, namely the Fubini-Jensen theorem on product measures is just enough, plus the scalar version of KMKA Lemma $\left(\mathscr{E}=\boldsymbol{C}^{1}\right)$. The probabilistic version of KMKA Lemma simply says that positive definite complex function is a covariance function of a zero mean Gaussian process. Let us recall that the random complex variable $\xi$ is zero mean Gaussian if $\xi \equiv 0$ or, if its complex characteristic function $\varphi_{\xi}(w)=M\left(e^{i \operatorname{Re} \bar{w} \xi}\right)$ ( $M \eta$ stands for the mean value of the random variable $\eta$ ) is of the form

$$
\varphi_{\xi}(w)=e^{-\frac{d}{4}|w|^{2}}=\frac{1}{\pi d} \int_{\boldsymbol{C}} e^{i \operatorname{Re} \bar{w} z} e^{-\frac{|z|^{2}}{d}} d \sigma(z),
$$

where $z=x+i y, d \sigma(z)=d x d y$ and $d>0-$ in fact $d=M|\xi|^{2}=$ the variance of $\xi$. The complex zero mean Gaussian process is the family $\xi_{x}(x \in X)$ of complex random variables over the common probability space and such one, that for every $n$, arbitrary $x_{1}, \ldots, x_{n} \in X, a_{1}, \ldots, a_{n} \in C$ the variable $\xi=\sum_{j \mid 1}^{n} \alpha_{j} \xi_{x_{j}}$ is a zero mean Gaussian variable.

We are now ready to formulate the KMKA probabilistic version following K. Ito (Jap. J. Math. 22 (1952), p. 63-86.) who proved it using scalar KMKA Lemma and Fubini-Jensens theorem:

Proposition 2.0. Let $(x, y) \rightarrow b_{x, y}$ be a complex positive definite scalar function $(x, y \in X)$. Then there is a Gaussian zero mean process $\xi_{x}$ such that $b_{x, y}=M\left(\xi_{x} \xi_{y}\right)$ for $x, y \in X$ (i.e. $b_{x, y}$ is the covariance function of $\left.\left\{\xi_{x}\right\}\right)$. 
Notice that the scalar version $\left(\mathscr{E}=C^{\mathbf{l}}\right)$ of KMKA Lemma implies its general form, for if $l_{x, y}(\cdot, \cdot)>>0$, then the scalar function

$$
(\{x, f\},\{y, g\}) \rightarrow l_{x, y}(f, g)
$$

is positive definite. This being established we get that $l_{x, y}(f, g)=(u(x, f)$, $\left.u(y, g)\right|_{K}$ and then check easily that $u(x, f)$ is linear in $f$. It remains to define $\bar{Y}(x) f=u(x, f)$. However, having in view Proposition 2.0 we get an extra profit, namely that $K$ can be chosen as a subspace of the space $L^{2}(P)$ where $P=(\Omega, \mathscr{B}, \mu)$ is a suitable probability space. This is a useful functional model of $K$ which we will exploit in the proof of the Górniak extension [7] of Naimark's [24] theorem on semispectral measures.

Proposition 2.1 (see [7]). Let $\mathscr{B}$ be a $\sigma$-field of subsets of the space $\Omega$. Suppose that $\mathscr{E}$ is a linear complex space and let the function $\mu(\sigma ; f, g)$ $\in C^{1} \quad(\sigma \in \beta ; f, g \in \mathscr{E})$ satisfy the following conditions:

(i) For every fixed $f, g \in \mathscr{E} \mu(\sigma ; f, g)$ is a complex measure on $\mathscr{B}$;

(ii) For every fixed $\sigma \in \mathscr{B} \mu(\sigma ; f, g)$ is bilinear in $f, g$;

(iii) $\mu(\sigma ; f, f) \geqslant 0$ for every $\sigma \in \mathscr{B}$ and for every $f \in \mathscr{E}$.

Then there is a complex Hilbert space $K$ and a spectral measure $E$ on $\mathscr{B}$, whose values are orthoprojections in $K$, and a linear operator $R: \mathscr{E} \rightarrow K$ such that

$\mu(\sigma ; f, g)=(E(\sigma) R f, R g)_{K} \quad$ for $\sigma \in \mathscr{B} ; f, g \in \mathscr{E}$.
The minimality obndition $K=\bigvee_{\sigma \in \mathscr{B}} E(\sigma) R \mathscr{E}$ determines $(K, E, R)$ up to unitary isomorphism, i.e. if $K^{\prime}=\bigvee_{\sigma \in \mathscr{S}} E^{\prime}(\sigma) R^{\prime}(\mathscr{E})$ where $E^{\prime}$ is a spectral measure on $\mathscr{B}$ which values are orthoprojections in $K^{\prime}$ and $R^{\prime}: \mathscr{E} \rightarrow K^{\prime}$ is a linear operator and moreover $\mu(\sigma ; f, g)=\left(E^{\prime}(\sigma) R^{\prime} f, R^{\prime} g\right)_{K^{\prime}}$ for $\sigma \in \mathscr{B}$; $f, g \in \mathscr{E}$, then there is a unitary map $U: K \rightarrow K^{\prime}$ such that $U R=R^{\prime}$ and $U E(\sigma)=E^{\prime}(\sigma) U$ for all $\sigma \in \mathscr{B}$.

Proof. Let $\sigma_{1}, \ldots, \sigma_{n} \in \mathscr{B}$ and $f_{1}, \ldots, f_{n} \in \mathscr{E}$ and take a positive measure $\mu(\sigma)=\sum_{j \mid 1}^{n} \mu\left(\sigma ; f_{j}, f_{j}\right)$. By the polarization formula and the Schwarz inequality, $\mu_{j k}(\cdot)=\mu\left(\cdot ; f_{j}, f_{k}\right)<<\mu$ for $j, k=1, \ldots, n$. Since by (iii) $\mu(\sigma ; g, g) \geqslant 0$, then taking $g=\sum_{j ! 1}^{n} a_{j} f_{j}$ we have that for $\sigma \in \mathscr{B}$

$$
\mu(\sigma ; g, g)=\sum_{j, k}\left(\int_{\sigma} h_{j k} d \mu\right) \alpha_{j} \bar{a}_{k} \geqslant 0
$$

where $h_{j k}=d \mu_{j, k} / d \mu$ (Randon-Nikodym derivative). Since $\sigma$ is arbitrary and $a$ 's contain a countable dense set, we derive that $\sum_{j, k \mid 1}^{n} h_{j, k}(\omega) a_{j} \bar{a}_{k l} \geqslant 0$ 
for almost all $\omega \in \Omega$ and arbitrary $\alpha_{1}, \ldots, \alpha_{n}$. By Schur's lemma and the trivial incquality $\sum_{j, k} \chi_{\sigma_{j} \cap \sigma_{k}} a_{j} \bar{a}_{k} \geqslant 0\left(\chi_{\sigma}\right.$ stands for the indicator function of the set $\sigma$ ) we get that

$$
\sum_{j, k} \mu\left(\sigma_{j} \cap \sigma_{l k} ; f_{j}, f_{k}\right)=\sum_{j, k} \int_{\sigma_{j} \cap \sigma_{k}} h_{j k} d \mu \geqslant 0 .
$$

It follows that the scalar function

$$
\left(\left\{\sigma^{\prime}, f\right\},\left\{\sigma^{\prime \prime}, g\right\}\right) \rightarrow \mu\left(\sigma^{\prime} \cap \sigma^{\prime \prime} ; f, g\right)
$$

is positive definite.

By Proposition 2.0, $\mu\left(\sigma^{\prime} \cap \sigma^{\prime \prime} ; f, g\right)=M\left(\xi\left(\sigma^{\prime}, f\right) \overline{\xi\left(\sigma^{\prime \prime}, g\right)}\right)$, where $\xi(\sigma, f)$ is a zero mean Gaussian process. As noticed before, $\xi(\sigma, f)$ is linear in $f$. We define $K$ as the closed span of $\xi(\sigma, f)(\sigma \in \mathscr{B}, f \in \mathscr{E})$ in $L^{2}(P)$, where $P=(\Omega, \mathscr{B}, \mu)$ is a suitable probability space and define $R f=\xi(\Omega, f)$ and $E(\sigma) R f=\xi(\sigma, f)=\xi(\sigma \cap \Omega, f)$, which proves, as easily seen, the first part of our assertion. The uniqueness statement has a standard proof (see [22], for instance) and we omit it.

3. Regulators of positive definite functions. Let $l=\left\{l_{x, y}(f, g)\right\}(x, y$ $\in X ; f, g \in \mathscr{E})$ be a positive definite function. Following Krein [14], Getoor [5], Masani [20] and Sz.-Nagy [33], we give an extract of thrir definitions.

Definition 3.0. Let $u: X \rightarrow X$ be a map. We say that $u$ is a weak regulator for $l>>0$ if the following condition holds true:

$$
\begin{gathered}
\sum_{j, k \mid 1}^{n} l_{x_{j} . x_{k}}\left(f_{j}, f_{k}\right)=0, \text { then } \\
\qquad \sum_{j, k \mid 1}^{n} l_{u\left(x_{j}\right), u\left(x_{k}\right)}\left(f_{j}, f_{k}\right)=0 .
\end{gathered}
$$

Definition 3.1. Suppose we are given the positive definite function $l=\left\{l_{x, y}(f, g)\right\}$ and the mapping $u: X \rightarrow X$. We say that $u$ is a regulator for $l$ if there is a finite positive constant $a(u)$ such that

$$
\sum_{j, k ! 1}^{n} l_{u\left(x_{j}\right), u\left(x_{k}\right)}\left(f_{j}, f_{k}\right) \leqslant a(u)^{2} \sum_{j, k \mid 1}^{n} l_{x_{j}, x_{k}}\left(f_{j}, f_{k}\right)
$$

for any $n=1,2, \ldots$, arbitrary $x_{1}, \ldots, x_{n} \in X, f_{1}, \ldots, f_{n} \in \mathscr{E}$.

The above definition originates from the boundedness condition in the Sz.-Nagy dilation theorem over involutory semi-groups - see [33].

We shall prove the following proposition essentially due to Getoor [5] (first part of assertion) and to Sz.-Nagy [33] and to Masani [20] and Kunze [15] (second part of assertion).

Proposition 3.0. Let $u: X \rightarrow X$ be a weak regulator of the positive definite function $l=\left\{l_{x, y}(f, g)\right\}$ with canonical representation $l_{x, y}(f, g)$ 
$=(Y(x) f, Y(y) g)_{K}$. Then there is the unique linear map $\tilde{\pi}(u)$ of the linear span $M \subset K$ of vector $Y(x) f(x \in X, f \in \mathscr{E})$ into itself and such that $\tilde{\pi}(u) Y(x) f$ $=Y(u(x))$ f for $x \in X$ and $f \in \mathscr{E}$. If $u$ is a regular for $l$ with the corresponding constant $a(u)$, then $\tilde{\pi}(u)$ extends by continuity in the unique way to the linear bounded operator $\pi(u)$ in $K$ and $\|\pi(u)\| \leqslant a(u)$.

Proof. The definition of a weak requlator implies that

$$
\text { if }\left\|\sum_{j \mid 1}^{n} Y\left(x_{j}\right) f_{j}\right\|_{K}^{2}=0 \text { then }\left\|\sum_{j \mid 1}^{n} Y\left(u\left(x_{j}\right)\right) f_{j}\right\|_{K}^{2}=0
$$

which proves the first part of our assertion by defining

$$
\tilde{\pi}(u) \sum_{j \backslash 1}^{n} Y\left(x_{j}\right) f_{j} \stackrel{\mathrm{df}}{=} \sum_{j \backslash 1}^{n} Y\left(u\left(x_{j}\right)\right) f_{j} .
$$

If (3.0) holds true then since for $y_{1}, \ldots, y_{n} \in X$

$$
\left\|\sum_{j \mid 1}^{n} Y\left(y_{j}\right) f_{j}\right\|_{K}^{2}=\sum_{j, k \mid 1}^{n} l_{y_{j}, y_{k}}\left(f_{j}, f_{k}\right)
$$

we have that

$$
\left\|\tilde{\pi}(u) \sum_{j \mid 1}^{n} Y\left(x_{j}\right) f_{j}\right\|_{K}^{2} \leqslant a(u)^{2}\left\|\sum_{j \mid 1}^{n} Y\left(x_{j}\right) f_{j}\right\|_{K^{r}}^{2}
$$

which completes the proof, because $M$ is dense in the minimal space $K$.

Notice that $\tilde{\pi}(u)$ corresponding to the weak regular $u$ of some $l$ need not be closable. To have an example we take $\mathscr{E}$ as an infinite dimensional Hilbert space and an unbounded linear operator $A$ defined all over $\mathscr{E}$. Such an $A$ cap be constructed with the help of a Hamel basis of $\mathscr{E}$. Now we take $X=\{0,1,2, \ldots\}$ and $l_{n, m}(f, g)=\left(A^{n} f, A^{m} g\right)$ and $u(n)=n+1$; $n, m \in X$. Since $A^{0} f=f$ for $f \in \mathscr{E}$, the equality defining $l$ is the canonical representation of $l$ with minimal space equal to $\mathscr{E}$. It is plain that $u$ is a weak regulator of $l$ and $\tilde{\pi}(u)=A$. The closed graph theorem shows that $\tilde{\pi}(u)$ is not closable. This is an observation of C. Ryll-Nardzewski (private communication) that for $\mathscr{E}=$ the space of complex polynomials in $t \in(-\infty,+\infty)$ and :

$$
l_{m, n}(f, g)=\int_{-\infty}^{+\infty} t^{m+n} f(t) \overline{g(t)} e^{-t^{2 / 2}} d t
$$

for $f, g \in \mathscr{E}$, the map $u(n)=n+1$ (we take $X=\{0,1,2, \ldots\}$ ) is a weak requlator for $l$ for which (3.0) fails.

Notice that here $K=L^{2}\left(\boldsymbol{R}^{1}, e^{-t^{2} / 2} d t\right)$.

13 - Annales Polonicl Mathematici XLII 
That $u$ is a weak regulator for $l$ is a trivial fact. On the other hand, since for $f(t) \equiv 1$

and

$$
l_{n+1, n+1}(f, f)=\int_{-\infty}^{+\infty} t^{2(n+1)} e^{-t^{2} / 2} d t=\sqrt{2 \pi} \prod_{k ; 1}^{n+1}(2 k-1)
$$

we have

$$
l_{n, n}(f, f)=\sqrt{2 \pi} \prod_{k \mid 1}^{n}(2 k-1)
$$

$$
\frac{l_{n+1, n+1}(f, f)}{l_{n, n}(f, f)}=2 n+1 \underset{n \rightarrow \infty}{\rightarrow} \infty
$$

which shows that (3.0) fails in this case.

The operator $\bar{\pi}(u)$ appearing in Proposition 3.0 is considered within the minimal space $K$. Two simple consequences of Proposition 3.0 should be noticed:

(3.1) If $u$ is a weak regulator for $l>>0$ and $u(X)=X$ then $\tilde{\pi}(u) M=M$ and consequently $\tilde{\pi}(u)^{*}$ is invertible.

(3.2) If $u$ is a regulator of $l>>$ and $u(X)=X$, then $\overline{\tilde{\pi}(u) K}=K$.

Let $\zeta_{l}^{w}$ be the totality of all weak regulators of $l>>0$ and $\zeta_{l}^{s}$ the totality of all regulators of $l>>0$. It is plain that if $u, v \in \zeta_{l}^{w(s)}$, then the composition $u v=u \circ v \in \zeta_{l}^{v(s)}$. Hence $\zeta_{l}^{w}$ and $\zeta_{l}^{8}$ are semigroups, the semigroup operation being the composition. The identity map $e$ of $X$ belongs to $\zeta_{l}^{w}$ and $\zeta_{l}^{8}$. It is easy to prove that:

(3.3) The map $u \rightarrow \tilde{\pi}(u)$ for $u \in \zeta_{l}^{w}(l>>0)$ is a unital representation of $\zeta_{l}^{20}$ into the algebra of linear maps of $M$ into $M$.

(3.4) The map $u \rightarrow \pi(u)$ for $u \in \zeta_{l}^{s}(l>>0)$ is a unital representation of $\zeta_{l}^{s}$ into $L(K)=$ the algebra of all linear bounded operators in the minimal space $K$ of the canonical representation of $l$.

The following proposition follows immediately from the proof of Proposition 3.0:

Proposition 3.1. If $l=\left\{l_{x, y}(f, g)\right\}>>0$ and $u: X \rightarrow X$ and $l_{x, y}(f, g)$ $=l_{u(x), u(v)}(f, g)$ for $x, y \in X ; g, f \in \mathscr{E}$, then $u$ is a regulator for $l$ and $\pi(u)$ is an isometry, which is unitary if $u(X)=X$.

CoROLLARY 3.0. Let $l>>0$ and denote by $G_{l}$ the totality of all one-to-one maps $u$ of $X$ onto $X$ such that $u$ leaves $l$ invariant, i.e. $l_{u(x), u(y)}(f, g)=l_{x, y}(f, g)$ for $x, y \in X ; f, g \in \mathbb{E}$. Then $G_{l}$ becomes a group with group operation equal to composition and the inverse of $u \in G_{l}$ equal to $u^{-1}$; $e$ is the unit of $G_{l}$. Proposition 3.1 implies that the map $u \rightarrow \pi(u)$ for $u \in G_{l}$ is a unitary representation of $G_{l}$ into $L(K)$, where $K$ is the corresponding to $l$ minimat space. 
This is essentially a generalization of Krein's [14] result and in fact takes place for any group of actions over $X$ leaving $l$ invariant. If $X$ is a group and $l_{x, y}(f, g)=l_{y^{-1}}(f, g)$, then $X$ is contained "naturally" in $G_{l}$ and the story reduces to Naimark's theorem [24], [22].

4. Conditional positive definiteness. This section deals with some extension of definition of scalar valued conditionally positive definite functions. Our partial scalar model here are definitions and theorems inclosed in the papers of Guichardet [8] and of Parthasarathy-Schmidt [27]. It should be mentioned that conditional positive definitness appears at the very begining in Schönberg's paper [32] and Krein [13], in connection with some concrete problems - see also Cuppens [3]. The crucial point of our considerations is that we deal with functions of the form $b$ $=\left\{b_{x, y}(f, g)\right\}$ where $f, g$ are in a linear space and $b_{x, y}(f, g)$ is bilinear in $f, g$. The classical case is just when $\mathscr{E}=C^{\mathrm{I}}$ or $\mathscr{E}=\boldsymbol{R}^{\mathbf{1}}$.

The central role is played by the structural function of $b_{x, y}(f, g)$. This function seems to be the proper concept in the study of conditionally positive definite functions. It appears naturally in the theory of second order stochastic processes with stationary increments. Our classical models here are Yaglom's papers [35], [36] and that ones of Masani [18], [19] - see enclosed references. The general theory is illustrated in our paper by discussing stationary functions of the form $b_{x-y}(f, g)$ with $x, y \in X$, where $X$ is a real separable Hilbert space, what is essentially related to Lery's-Khintchine formula and helixes and some special unitary representations of the additive group of $X$. We should however point out that our general results do not require any kind of hermitian symmetry of functions in consideration and may be applied for instance to functions on semi-groups.

In all what follows $\mathscr{E}$ is a linear complex space and $X$ stands for some abstract fixed set. Recall that the complex valued function $b_{x, y}(x, y \in X)$ is called conditionally positive definite if for every $n=1,2, \ldots$ and any $x_{1}, \ldots, x_{n} \in X$ and $\hat{a_{1}}, \ldots, a_{n} \in C$ such that $\sum_{j \mid 1}^{n} a_{j}=0$, the inequality $\sum_{j, k \mid 1}^{n} b_{x_{j}, x_{k}} a_{j} \tilde{a}_{k} \geqslant 0$ holds true.

It is proved in [27] that the following condition holds true:

(4.0) If $b_{x, y}(x, y \in X)$ is a scalar function which is hermitian symmetric, i.e. $b_{x, y}=\overline{b_{y, x}}$, then the following conditions are equivalent:

(i) $b_{x, y}$ is conditionally positive definite.

(ii) For every fixed $\tau>0$ the function $s_{x, y}^{\tau}=e^{\tau b x, \nu}$ is positive definite.

Suppose once for all in what follows that $b=\left\{b_{x, y}(f, g)\right\}$ is a family of bilinear forms in $f, g \in \mathscr{E}$. The following definition is now in order. 
DeFINITION 4.0. We say that $b=\left\{b_{x, y}(f, g)\right\}$ is weakly conditionally positive definite if for every fixed $f \in \mathscr{E}$ the scalar function $(x, y) \rightarrow b_{x, y}(f, f)$ is conditionally positive definite.

DEFINITION 4.1. We say that the function $b=\left\{b_{x, y}(f, g)\right\}$ is conditionally positive definite if for every $n=1,2,3, \ldots$, arbitrary $x_{1}, \ldots, x_{n} \in X$ and $f_{1}, \ldots, f_{n} \in \mathscr{E}$ such that $\sum_{j \mid 1}^{n} f_{j}=0$, the inequality $\sum_{j, k \mid 1}^{n} b_{x_{j}, x_{k}}\left(f_{j}, f_{k}\right) \geqslant 0$
holds true.

It is plain that if $b$ is conditionally positive definite then $b$ is weakly conditionally positive definite.

EXAMPLE 4.0. Let $\mathscr{E}$ be a complex Hilbert space and let $B(x, y)$ $(x, y \in X)$ be a family of linear bounded operators in $\mathscr{E}$. If for every $\tau>0$ the function $c_{x, y}^{\tau}(f, g)=\left(e^{\tau B(x, y)} f, g\right)$ is positive definite, then the function $b_{x, y}(f, g)=(B(x, y) f, g)$ is conditionally positive definite. Indeed, if $\sum_{j \mid 1}^{n} f_{j}=0, \tau>0$ and $\varphi(\tau) \stackrel{\text { df }}{=} \sum_{j, k \mid 1}^{n}\left(e^{\tau B\left(x_{j}, x_{k}\right)} f_{j}, f_{k}\right)$, then $\varphi(\tau) \geqslant 0=\varphi(0)$ which implies that

$$
\sum_{j, k \mid 1}^{n}\left(B\left(x_{j}, x_{k}\right) f_{j}, f_{k}\right)=\lim _{\tau \rightarrow 0+} \frac{\varphi(\tau)-\varphi(0)}{\tau} \geqslant 0 .
$$

We have next the following proposition.

Proposition 4.0. Suppose we are given the function $b=\left\{b_{x, y}(f, g)\right\}$. Then the following conditions are equivalent:

(4.1) $b$ is conditionally positive definite.

(4.2) For every $x_{0} \in X$ the function

$$
c_{x, y}^{x_{0}}(f, g) \stackrel{\text { df }}{=} b_{x, y}(f, g)-b_{x, x_{0}}(f, g)-b_{x_{0}, y}(f, g)+b_{x_{0}, x_{0}}(f, g)
$$

is positive definite.

Proof. Suppose that (4.1) holds true. We take $x_{1}, \ldots, x_{n} \in X$ and $f_{1}, \ldots, f_{n} \in \mathscr{E}$ and define $f_{0}=-\sum_{j \mid 1}^{n} f_{j}$ and fix an $x_{0} \in X$. Since (4.1) holds true by assumption, we get that

Now

$$
a \stackrel{\text { dt }}{=} \sum_{j, k \mid 0}^{n} b_{x_{j}, x_{k}}\left(f_{j}, f_{k}\right) \geqslant 0
$$

$$
\begin{aligned}
a & =\sum_{j \mid 0}^{n} \sum_{k \mid 0}^{n} b_{x_{j}, x_{k}}\left(f_{j}, f_{k}\right)=\sum_{k \mid 0}^{n} b_{x_{0}, x_{k}}\left(f_{0}, f_{k}\right)+\sum_{j \mid 1}^{n} \sum_{k \mid 0}^{n} b_{x_{j}, x_{k}}\left(f_{j}, f_{k}\right) \\
& =\sum_{k \mid 0}^{n} b_{x_{0}, x_{k}}\left(-\sum_{j \mid 1}^{n} f_{j}, f_{k}\right)+\sum_{j \mid 1}^{n}\left[b_{x_{j}, x_{0}}\left(f_{j}, f_{0}\right)+\sum_{k \mid 1}^{n} b_{x_{j}, x_{k}}\left(f_{j}, f_{k}\right)\right]
\end{aligned}
$$




$$
\begin{aligned}
= & -b_{x_{0}, x_{0}}\left(\sum_{j \mid 1}^{n} f_{j},-\sum_{k \mid 1}^{n} f_{k}\right)+\sum_{k \mid 1}^{n} b_{x_{0}, x_{k}}\left(-\sum_{j \mid 1}^{n} f_{j}, f_{k}\right)+\sum_{j \mid 1}^{n} b_{x_{j}, x_{0}}\left(f_{j}, f_{0}\right)+ \\
= & \sum_{j, k \mid 1}^{n} \sum_{j \mid 1}^{n} b_{x_{0}, x_{0}}\left(f_{j}, f_{k}\right)-\sum_{x_{j}, x_{k}}\left(f_{j}, f_{k}\right) \\
& -\sum_{j \mid 1}^{n} \sum_{j \mid 1}^{n} b_{x_{0}, x_{k}}\left(f_{j}, f_{k}\right)- \\
& \sum_{x_{j}, x_{0}}\left(f_{j}, f_{k}\right)+\sum_{j \mid 1}^{n} \sum_{k \mid 1}^{n} b_{x_{j}, x_{k}}\left(f_{j}, f_{k}\right)=\sum_{j, k \mid 1}^{n} e_{x_{j}, x_{k}}^{x_{0}}\left(f_{j}, f_{k}\right) \geqslant 0 .
\end{aligned}
$$

We just proved that (4.1) implies (4.2). Suppose now that (4.2) holds true. Suppose $x_{0}, x_{1}, \ldots, x_{n} \in X$ and $\sum_{j \mid 0}^{n} f_{j}=0$, i.e. $f_{0}=-\sum_{j \mid 0}^{n} f_{j}$. We have
to show that

$$
c^{\prime}=\sum_{j, k \mid 0}^{n} b_{x_{j}, x_{k}}\left(f_{j}, f_{k}\right) \geqslant 0 .
$$

Our assumption implies that

$$
\begin{aligned}
c & =\sum_{j, k \mid 1}^{n} c_{x_{j}, x_{k}}^{x_{0}}\left(f_{j}, f_{k}\right) \\
& =\sum_{j, k \mid 1}^{n}\left(b_{x_{j}, x_{k}}\left(f_{j}, f_{k}\right)-b_{x_{0}, x_{k}}\left(f_{j}, f_{k}\right)-b_{x_{j}, x_{0}}\left(f_{j}, f_{k}\right)+b_{x_{0}, x_{0}}\left(f_{j}, f_{k}\right)\right) \geqslant 0 .
\end{aligned}
$$

Since

$$
\sum_{j, k \mid 1}^{n} b_{x_{0}, x_{0}}\left(f_{j}, f_{k}\right)=b_{x_{0}, x_{0}}\left(-\sum_{j \mid 1}^{n} f_{j},-\sum_{k \mid 1}^{n} f_{k}\right)=b_{x_{0}, x_{0}}\left(f_{0}, f_{0}\right)
$$

we conclude that

$$
c=\sum_{j, k \mid 1}^{n}\left[b_{x_{j}, x_{k}}\left(f_{j}, f_{k}\right)-b_{x_{0}, x_{k}}\left(f_{j}, f_{k}\right)-b_{x_{j}, x_{0}}\left(f_{j} ; f_{k}\right)\right]+b_{x_{0}, x_{0}}\left(f_{0}, f_{0}\right) .
$$

On the other hand,

$$
\begin{aligned}
& -\sum_{j \mid 1}^{n} \sum_{k \mid 1}^{n} b_{x_{0}, x_{k}}\left(f_{j}, f_{k}\right)=\sum_{k \mid 1}^{n} b_{x_{0}, x_{k}}\left(f_{0}, f_{k}\right), \\
& -\sum_{j \mid 1}^{n} \sum_{k \mid 1}^{n} b_{x_{j}, x_{0}}\left(f_{j}, f_{k}\right)=\sum_{j \mid 1}^{n} b_{x_{j}, x_{0}}\left(f_{j}, f_{0}\right) .
\end{aligned}
$$


It follows that

$$
\begin{aligned}
c & =\sum_{k \mid 1}^{n} \sum_{j \mid 1}^{n} b_{x_{j}, x_{k}}\left(f_{j}, f_{k}\right)+\sum_{k \mid 1}^{n} b_{x_{0}, x_{k}}\left(f_{0}, f_{k}\right)+ \\
& \quad+\sum_{j \mid 1}^{n} b_{x_{j}, x_{0}}\left(f_{j}, f_{0}\right)+b_{x_{0}, x_{0}}\left(f_{0}, f_{0}\right) \\
= & \sum_{k \mid 1}^{n}\left[\sum_{j \mid 1}^{n} b_{x_{j}, x_{k}}\left(f_{j}, f_{k}\right)+b_{x_{0}, x_{k}}\left(f_{0}, f_{k}\right)\right]+\sum_{j \mid 0}^{n} b_{x_{j}, x_{0}}\left(f_{j}, f_{0}\right) \\
= & \sum_{k \mid 1}^{n}\left[\sum_{j \mid 0}^{n} b_{x_{j}, x_{k}}\left(f_{j}, f_{k}\right)\right]+\sum_{j \mid 0}^{n} b_{x_{j}, x_{0}}\left(f_{j}, f_{0}\right) \\
= & \sum_{k \mid 0}^{n}\left[\sum_{j \mid 0}^{n} b_{x_{j}, x_{k}}\left(f_{j}, f_{k}\right)\right]=c^{\prime} \geqslant 0 \quad \text { because } c \geqslant 0 .
\end{aligned}
$$

Suppose that the function $b=\left\{b_{x, y}(f, g)\right\}$ is conditionally positive definite. We take $x_{0} \in X$ and conclude that the function $c_{x, y}^{x_{0}}(f, g)$ is positive definite. Let $c_{x, v}^{x_{0}}(f, g)=\left(Y_{x_{0}}(x) f, Y_{x_{0}}(y) g\right) \kappa_{x_{0}}$ be the canonical representation of $c_{x, y}^{x_{0}}$. Then $K_{x_{0}}$ is minimal, i.e. $K_{x_{0}}=\bigvee Y_{x \in X} Y_{x_{0}}(x) \mathscr{E}$. We say then that $c_{x, y}^{x_{0}}, K_{x_{0}}, Y_{x_{0}}(\cdot)$ are associated with $x_{0}$.

Proposition 4.1. Let $b=\left\{b_{x, y}(f, g)\right\}$ be a conditionally positive definite function. Suppose $c_{x, y}^{x_{0}}, K_{x_{0}}, Y_{x_{0}}$ are associated with $x_{0}$. Then

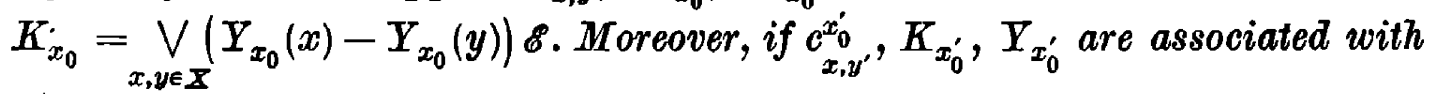
$x_{0}^{\prime}$, then there exists the unique unitary map $V_{x_{0}, x_{0}^{\prime}}: K_{x_{0}} \rightarrow K_{x_{0}^{\prime}}$ such that

$$
\nabla_{x_{0}, x_{0}^{\prime}}\left(Y_{x_{0}}(x)-Y_{x_{0}}(y)\right) f=\left(Y_{x_{0}^{\prime}}(x)-Y_{x_{0}^{\prime}}(y)\right) f
$$

for all $x, y \in X$ and $f \in \mathscr{E}$.

Proof. Notice that $c_{x_{0}, x_{0}}^{x_{0}}(f, g)=\left(Y_{x_{0}}\left(x_{0}\right) f, Y_{x_{0}}\left(x_{0}\right) g\right)_{K_{x_{0}}}=0$ which shows that $Y_{x_{0}}\left(x_{0}\right)=0$. It follows that

$$
K_{x_{0}} \subset \bigvee_{x, y \in X}\left(Y_{x_{0}}(x)-Y_{x_{0}}(y)\right) \mathscr{E}
$$

Notice now that for $x, y, x^{\prime}, y^{\prime} \in X$ and $f, g \in \mathscr{E}$

$$
\begin{aligned}
\left(\left(Y_{x_{0}}(x)-Y_{x_{0}}(y)\right) f\right. & \left.\left(Y_{x_{0}}\left(x^{\prime}\right)-Y_{x_{0}}\left(y^{\prime}\right)\right) g\right)_{K_{x_{0}}}=\Delta \\
& =c_{x, x^{\prime}}^{x_{0}}(f, g)-c_{x, y^{\prime}}^{x_{0}}(f, g)-c_{y, x^{\prime}}^{x_{0}}(f, g)+c_{y, y^{\prime}}^{x_{0}}(f, g) \\
& =b_{x, x^{\prime}}(f, g)-b_{x, y^{\prime}}(f, g)-b_{y, x^{\prime}}(f, g)+b_{y, y^{\prime}}(f, g) .
\end{aligned}
$$

Since the last term of the above equality does not depend on $x_{0}$, we conclude that

$$
\Delta=\left(\left(Y_{x_{0}^{\prime}}(x)-Y_{x_{0}^{\prime}}(y)\right) f,\left(Y_{x_{0}^{\prime}}\left(x^{\prime}\right)-Y_{x_{0}^{\prime}}\left(y^{\prime}\right)\right) g\right)_{K_{x_{0}}}
$$


which, since $K_{x_{0}\left(x_{0}^{\prime}\right)}=\underset{x, y \in X}{\bigvee}\left(Y_{x_{0}\left(x_{0}^{\prime}\right)}(x)-Y_{x_{0}\left(x_{0}^{\prime}\right)}(y)\right) \mathscr{E}$, proves the rest of our
assertion.

Remark 4.0. It is plain that the unitary maps $V_{u, v}(u, v \in X)$ of the above proposition satisfy the hemigroup equation, i.e. $V_{u, v} V_{z, u}$ $=V_{z, v}$ for $u, v, z \in X$.

Following the terminology of the theory of stochastic processes we introduce the following definition:

DefInITION 4.2. Let $b=\left\{b_{x, y}(f, g)\right\}$ be an arbitrary family of bilinear forms in $f, g \in \mathscr{E}$. The function

$$
d_{(x, y) ;\left(x^{\prime}, y^{\prime}\right)}(f, g)=b_{x, x^{\prime}}(f, g)-b_{x, y^{\prime}}(f, g)-b_{y, x^{\prime}}(f, g)+b_{y, y^{\prime}}(f, g)
$$

is called the structural function of $b$.

The structural function $d$ of $b$ is a function defined on $(X \times X) \times(X \times$ $\times X)$, whose values are bilinear forms on $\mathscr{E}$. If $b$ is conditionally positive definite then, by using Proposition 4.1

$$
\begin{aligned}
d_{(x, y) ;\left(x^{\prime}, y^{\prime}\right)}(f, g)= & \left(Z_{x_{0}}(x, y) f, Z_{x_{0}}\left(x^{\prime}, y^{\prime}\right) g\right)_{K_{x_{0}}} \\
& \text { where } \quad Z_{x_{0}}(u, v) f=\left(Y_{x_{0}}(u)-Y_{x_{0}}(v)\right) f
\end{aligned}
$$

which, since $K_{x_{0}}=\bigvee_{x, y \in \mathscr{E}} Z_{x_{0}}(x, y) \mathscr{E}$, proves that $d$ is positive definite and (4.4) is the canonical representation of $d$. Moreover, when varying $x_{0}$ over $X$ we get a family of such representations via the functions $c_{x, y}^{x_{0}}$ and the operators $V_{x_{0}, x_{0}^{\prime}}$ establish the suitable unitary equivalence of representations.

Proposition 4.2. Let $b=\left\{b_{x, y}(f, g)\right\}$ be a family of bilinear forms of $f, g \in \mathscr{E} . b$ is conditionally positive definite if and only if the structural function $d$ of $b$ is positive definite.

Proof. All we need to prove is that if $d>>0$, then $b$ is conditionally positive definite. If $d>0$ then for any $n, x_{j}, y_{j} \in X \quad(j=1, \ldots, n)$ and $f_{1}, \ldots, f_{n} \in \mathscr{E}$ the inequality

$$
\sum_{j, k\} 1}^{n} d_{\left(x_{j}, y_{j}\right) ;\left(x_{k}, y_{k}\right)}\left(f_{j}, f_{k}\right) \geqslant 0
$$

holds true. Let us take $y_{1}=y_{2}, \ldots, y_{n}=y_{0}$. Since $d_{\left(x_{j}, y_{0}\right),\left(x_{k}, y_{0}\right)}(f, g)$ $=c_{x_{j}, x_{k}}^{\nu_{0}}(f, g)$ and $y_{0}$ is arbitrary, Proposition 4.0 implies that $b$ is conditionally positive definite.

Suppose now that $b=\left\{b_{x, y}(f, g)\right\}$ is a function such that for some $x_{0} \in X$ the function

$$
c_{x, y}^{x_{\theta}}(f, g) \stackrel{d f}{=} b_{x, y}(f, g)-b_{x_{0}, y}(f, g)-b_{x, x_{0}}(f, g)+b_{x_{0}, x_{0}}(f, g)
$$

is positive definite, and $c_{x, y}^{x_{0}}(f, g)=\left(Y^{0}(x) f, Y^{0}(y) g\right)_{K}$ is its canonical representation. Using formula (4.3) we find out that the structural function 
$d$ of $b$ is of the following form:

$$
d_{\left(x, y^{\prime}\right) ;\left(x^{\prime}, y^{\prime}\right)}(f, g)=\left(\left(Y^{0}(x)-Y^{0}(y)\right) f,\left(Y^{0}\left(x^{\prime}\right)-Y^{0}\left(y^{\prime}\right)\right) g\right)_{K} \cdot
$$

Consequently, $d$ is then positive definite. Having in view Propositions 4.0 and 4.2 we arrive to the following theorem:

THEOREM 4.0. Let $b=\left\{b_{x, y}(f, g)\right\}$ be a family of bilinear forms. Then the following conditions are equivalent:

(i) $b$ is conditionally positive definite.

(ii) For every $x_{0} \in X$ the function

$$
c_{x, y}^{x_{0}}(f, g)=b_{x, y}(f, g)-b_{x_{0}, y}(f, g)-b_{x, x_{0}}(f, g)+b_{x_{0}, x_{0}}(f, g)
$$

is positive definite.

(iii) The structural function $d$ of $b$ is positive definite. $x_{0} \in X$.

(iv) The function $c_{x, y}^{x_{0}}$ defined as in (ii) is positive definite for some

Theorem 4.0 as well all we said before and will say later on, points out the crucial role of structural function in a rather general circumstances, which seems to be overlooked in the general theory.

If $Y(x): \mathscr{E} \rightarrow K$ are linear maps for $x \in X$ then the function

$$
d_{(x, y) ;\left(x^{\prime}, y^{\prime}\right)}(f, g)=\left((Y(x)-Y(y)) f,\left(Y\left(x^{\prime}\right)-Y\left(y^{\prime}\right)\right) g\right)_{K}
$$

is the structural function of the function $b_{x, y}(f, g) \stackrel{d f}{=}(Y(x) f, Y(y) g)_{K}$ and $b_{x, y}(\cdot, \cdot)$ is positive definite and vice versa, if $b_{x, y}(\cdot, \cdot)>>0$ has the canonical representation $b_{x, y}(f, g)=(Y(x) f, Y(y) g)_{K}$ then $d$ as defined above via $Y(\cdot)$ is the structural function of $b$. Our goal however is that structural functions are positive definite for functions which are conditionally positive definite, hence for a class of functions essentially larger than the class of positive definite functions.

We are now in position to apply the general technique of using regulators to structural functions.

Definition 4.3. Let $b=\left\{b_{x, y}(f, g)\right\}$ be a conditionally positive function and $d_{(x, y),\left(x^{\prime}, y^{\prime}\right)}(f, g)$ its structural function. The mapping $u$ : $X \rightarrow X$ is called a weak regulator for $b$ if the mapping $\tilde{u}: X \times X \rightarrow X \times X$ defined as $\vec{u}(x, y)=\{u(x), u(y)\}$ for $x, y \in X$ is a weak regulator for $d$.

Proposition 3.0 and Proposition 4.1 (with corresponding notation) imply the following:

Proposition 4.3. Let $\iota: X \rightarrow X$ be a weak regulator of the conditionally positive definite function $b=\left\{b_{x, y}(f, g)\right\}$ and let $c_{x, y}^{x_{0}}, K_{x_{0}}, \vec{Y}_{x_{0}}$ be associated with $x_{0}$. Then there is the unique linear map $\tilde{\pi}_{x_{0}}(u)$ of the manifold $M_{x_{0}} \subset K_{x_{0}}$ $=\bigvee_{x, y \in X}\left(Y_{x_{0}}(x)-Y_{x_{0}}(y)\right) \mathscr{E}$ spanned by vectors $\left(Y_{x_{0}}(x)-Y_{x_{0}}(y)\right) f$ such that $\tilde{\pi}_{x_{0}}(u)\left(Y_{x_{0}}(x)-Y_{x_{0}}(y)\right) f=\left(Y_{x_{0}}(u(x))-Y_{x_{j}}(u(y))\right) f \quad$ for $\quad x, y \in X, \quad f \in \mathscr{E}$. 
The map $V_{x_{0}, x_{0}^{\prime}}$ establishes the unitary equivalence of $\tilde{\pi}_{x_{0}}(u)$ and $\tilde{\pi}_{x_{0}^{\prime}}(u)$ for any $x_{0}, x_{0}^{\prime} \in X$.

Remark 4.1. When keeping $x_{0}$ fixed we conclude that the map $u \rightarrow \tilde{\pi}_{x_{0}}(u)$, which generalizes the idea of cocycle of first order ([27], p. 11) is a unital representation of the semigroup of weak regulators (the semigroup operation being the composition of maps) into the algebra of linear maps of $M_{x_{0}}$ into itself.

The final part of Proposition 3.0 implies the following theorem:

THEOREM 4.1. Let the structural function $d$ of the conditionally positive definite function $b$ satisfy the following condition for the mapping $u: X \rightarrow X$ :

$$
\sum_{j, k \mid 1}^{n} d_{\left(u\left(x_{j}\right), u\left(y_{j}\right)\right) ;\left(u\left(x_{k}, u\left(y_{k}\right)\right)\right.}\left(f_{j}, f_{k}\right) \leqslant a(u)^{2} \sum_{j, k \mid 1}^{n} d_{\left(x_{j}, y_{j}\right) ;\left(x_{k}, y_{k}\right)}\left(f_{j}, f_{k}\right)
$$

for any $n$, arbitrary $x_{j}, y_{j} \in X(j=1, \ldots, n)$ and $f_{1}, \ldots, f_{n} \in \mathscr{E}$ with some finite positive constant $a(u)$ independent of $n, x$-es, $y$-es and $f$-es. Then $u$ is a weak regulator for $b$ and, using notation of Proposition 4.3 for every $x_{0} \in X$ the mapping $\tilde{\pi}_{x_{0}}(u)$ extends in the unique way to the linear bounded operator $\pi_{x_{0}}(u)$ on $K_{x_{0}}$ such that $\left\|\pi_{x_{0}}(u)\right\| \leqslant a(u)$. For any $x_{0}, x_{0}^{\prime}$ the operators $\pi_{x_{0}}(u)$ and $\pi_{x_{0}^{\prime}}(u)$ are unitarily equivalent via the unitary isomorphism $V_{x_{0}, x_{0}^{\prime}}$.

The above theorem is a genc ralization of a theorem of von NeumannSchönberg [25] - see also Masani [18], who considered the helixes, which correspond within our general frames to the case when $\mathscr{E}=C^{1}, X$ is a group equal to $\boldsymbol{R}^{n}$ and $u(x)=u+x$ as well in (4.5) we have the equality with $a(u)=1$ - the corresponding $\pi_{x_{0}}(u)$ is then a unitary operator. Similar situation is studied generally in more detail in the next section. $\pi_{x_{0}}(u)$ can be treated as a first order cocycle ([8], [27]).

5. Invariant structural functions. Let $b=\left\{b_{x, y}(f, g)\right\}$ (arbitrary) be given and let $d$ be the structural function of $b$. We say that for $u$ : $X \rightarrow X, d$ is $u$-invariant if

$$
d_{(u(x), u(y)) ;\left(u\left(x^{\prime}\right), u\left(y^{\prime}\right)\right)}(f, g)=d_{(x, y) ;\left(x^{\prime}, y^{\prime}\right)}(f, g)
$$

for all $x, y, x^{\prime}, y^{\prime} \in X$ and $f, g \in \mathscr{E}$.

The set $G_{d}$ of all one-to-one maps $u$ of $X$ onto for which $d$ is $u$-invariant is a group, the group operation being composition of the maps, $u^{-1}=$ inverse map of $u$ and $e=$ the identity map of $X$ is the unit of $G_{d}$.

Using freely the notation of the previous section we formulate the following theorem:

THEOREM 5.0. Let $b=\left\{b_{x, y}(f, g)\right\}$ be conditionally positive definite and $d$ the structural function of $b$. Let us take $c_{x, y}^{x_{0}}, K_{x_{0}}, Y_{x_{0}}(\cdot)$ associated to $x_{0}$. Then the mapping $G_{d}: u \rightarrow \pi_{x_{0}}(u)$ is a unitary representation of $G_{d}$; $\pi_{x_{0}}(\cdot), \pi_{x_{0}^{\prime}}$ are unitary equivalent via $V_{x_{0}, x_{0}^{\prime}}$. 
Proof. Since for $u \in G_{d}$ we have in (4.5) the equality with $a(u)=1$ and $u(X)=X$, then $\pi_{x_{0}}(u)$ is an isometry and since obviously $\pi_{x_{0}}(u) M_{x_{0}}$ $=M_{x_{0}}, \pi_{x_{0}}(u)$ is in fact unitary. The rest of the assertion follows from (3.4) when applied to $d$ in place of $l, X$ being replaced by $X \times X$.

Remark 5.0. The above proof can be reduced simply to using Proposition 3.1 to $l=d$ with $X$ replaced by $X \times X$.

Remark 5.1. The above theorem can be treated as the generalization of von Neumann-Schönberg theorem [25], the structural function $d$ being corresponding to the "generalized" helix ("screw line") $Y_{x_{0}}(x)$ and $\left\{\pi_{x_{0}}(u)\right\}$ the shift unitary group. In particular, if $X$ is a group itself with multiplicatively written group operation, then $X \subset G_{d}$ by identifying $u \in X$ with the map $u(x)=u x$, and $\left\{\pi_{x_{0}}(u)\right\}$ restricted to $u \in X$ is a unitary representation of $X$.

Remark 5.2. The set of maps $u: X \rightarrow X$ is a unital semigroup with semigroup operation being the composition. Let $G_{d}^{+}$be the subsemigroup of this semigroup of all $u: X \rightarrow X$ which leave $d$ invariant. Then the map $u \rightarrow \pi_{x_{0}}(u)$ is a semigroup isometric representation of $G_{d}^{+}$.

Following Parthasarathy-Schmidt [27] we introduce the following definition :

Definition 5.0. Let $b=\left\{b_{x, y}(f, g)\right\}$ be given and the map $u: X \rightarrow X$. We say that $b$ is u-affine invariant if there are bilinear in $f, g$ forms $\beta_{u}(x ; f, g)(x \in X)$ such that

$$
b_{u(x), u(y)}(f, g)=b_{x, y}(f, g)+\beta_{u}(x ; f, g)+\beta_{u}(y ; f, g)
$$

for all $x, y \in X$ and $f, g \in \mathscr{E}$. If $\beta_{u} \equiv 0$ we say that $b$ is $u$-invariant.

Notice that if $b$ is $u$-affine invariant then the structural function $d$ of $b$ is $u$-invariant. Indeed,

$$
\begin{aligned}
& d_{(u(x), u(y)) ;\left(u\left(x^{\prime}\right), u\left(v^{\prime}\right)\right)}(f, g)=b_{u(x), u\left(x^{\prime}\right)}(f, g)- \\
& \quad-b_{u(x), u\left(y^{\prime}\right)}(f, g)-b_{u(y), u\left(x^{\prime}\right)}(f, g)+b_{u(y), u\left(v^{\prime}\right)}(f, g) \\
& =b_{x, x^{\prime}}(f, g)+\beta_{u}(x ; f, g)+\beta_{u}\left(x^{\prime} ; f, g\right)-b_{x, y^{\prime}}(f, g)- \\
& \quad-\beta_{u}(x ; f, g)-\beta_{u}\left(y^{\prime} ; f, g\right)-b_{y, x^{\prime}}(f, g)-\beta_{u}(y ; f, g)-\beta_{u}\left(x^{\prime} ; f, g\right)+ \\
& \quad+b_{y, y^{\prime}}(f, g)+\beta_{u}(y ; f, g)+\beta_{u}\left(y^{\prime} ; f, g\right)=d_{(x, y) ;\left(x^{\prime}, v^{\prime}\right)}(f, g) .
\end{aligned}
$$

Using previous notation we arrive via Proposition 4.3 to the following theorem which generalizes Theorem 3.4 [27]:

THEOREM 5.1. Let $b=\left\{b_{x, y}(f, g)\right\}$ be an $u$-affine invariant conditionally positive definite function and let $e_{x, y}^{x_{0}}, K_{x_{0}}, Y_{x_{0}}$ be associated to $x_{0} \in X$. Then the map $\pi_{x_{0}}(u)$ is an isometry. If $u(X)=X$, then $\pi_{x_{0}}(u)$ is unitary.

Remark 5.3. We know that $\pi_{x_{0}}(u) Y_{x_{0}}(x) f=\left(Y(u(x))-Y\left(u\left(x_{0}\right)\right)\right) f$ for $x \in X, f \in \mathscr{E}$. Hence $\pi_{x_{0}}(u)$ can be called an isometric (unitary resp. if 
$u(X)=X$ ) operator valued first order cocycle related to $b$. The choice of $x_{0}$ is in fact inessential having in view the Proposition 4.1. Taking $\beta_{u} \equiv 0$ we get Theorem 5.0 for suitable $u$ as well the statement of Remark 5.2.

The special case of Theorem 5.0 should be now mentioned. It is in fact a reformulation of a special case of Remark 5.1. Suppose namely that $X$ is a multiplicatively written group with unit $e$ and consider the functions $b_{x}(f, g)$ indexed by $x \in X$ and such that $b_{x}(f, g)$ is a bilinear form in $f, g \in \mathscr{E}$. We say that $b_{x}(f, g)$ is conditionally positive definite on $X$ if the function $\tilde{b}_{x, y}(f, g) \stackrel{\text { df }}{=} b_{y^{-1}}(f, g)$ is conditionally positive definite. Now, to every $u \in X$ we have the mapping $u: X \rightarrow X$ defined on $X$, such that $u(X)=X$ and since $b_{y^{-1} x}(f, g)=b_{(u \nu)^{-1}(u x)}(f, g)$ for $x, y \in X$ and $f, g \in \mathscr{E}$ the structural function $\tilde{d}$ of $\tilde{b}$ is $u$-invariant. Now, combining Theorem 5.0 with Remark 5.1 we arrive to the following theorem:

THEOREM 5.2. Let $X$ be a multiplicative group with unit $e$ and let the function $b=\left\{b_{x}(f, g)\right\}$ be a conditionally positive definite function on $X$. Suppose that $b_{e}(f, g)=0$ for $f, g \in \mathscr{E}$ and let

$$
c_{y^{-1} x}^{e}(f, g)=b_{y^{-1} x}(f, g)-b_{x}(f, g)-b_{y^{-1}}(f, g), \quad K_{e}, Y_{e}(\cdot)
$$

be associated to e. The map $X: u \rightarrow \pi_{e}(u)$ is a unitary representation of $X$ into $L\left(K_{e}\right)$ and the formula $\pi_{e}(u) Y_{e}(x)=Y_{e}(u x)-Y_{e}(x)$ defines a unitary valued operator first order cocycle.

Remark 5.4. Suppose the assumptions of Theorem 5.2 hold true and additionally the structural function of $\tilde{b}$ is of the form

$$
d_{(x, y) ;(u, v)}(f, g)=z\left(y^{-1} x, v^{-1} u ; f, g\right) .
$$

Using notation of Theorem 5.2 we have that

$$
d_{(x, y) ;(u, v)}(f, g)=\left(\left(Y_{e}(x)-Y_{e}(y)\right) f,\left(Y_{e}(u)-Y_{e}(v)\right) g\right)_{K_{0}} .
$$

Notice that $\pi_{e}(u)\left(Y_{e}(x)-Y_{e}(y)\right) h=\left(Y_{e}(u x)-Y_{e}(u y)\right) h$ for $u, x, y \in X$ and $h \in \mathscr{E}$. By (5.0)

$$
\begin{aligned}
a & =\left\|\left(Y_{e}(u x)-Y_{e}(u y)\right) f-\left(Y_{e}(x)-Y_{e}(y)\right) f\right\|_{K_{e}}^{2} \\
& =d_{(u x, u y) ;(u x, u y)}(f, f)+d_{(x, y) ;(x, y)}(f, f)-2 \operatorname{Re} d_{(u x, u y) ;(x, y)}(f, f) \\
& =2 z\left(y^{-1} x, y^{-1} x ; f, f\right)-2 \operatorname{Re} z\left(y^{-1} x, y^{-1} x ; f, f\right) .
\end{aligned}
$$

But $d$ is hermitian symmetric as a positive definite function, that is,

$$
\overline{d_{(x, y) ;(u, v)}(f, g)}=d_{(u, v) ;(x, v)}(g, f) \quad(x, y, u, v \in X ; f, g \in \mathscr{E}) .
$$

It follows from (5.0) that

$$
\overline{z\left(y^{-1} x, y^{-1} x ; f, f\right)}=z\left(y^{-1} x, y^{-1} x ; f, f\right)
$$


which proves that $a=0$. Since $K_{e}$ is spanned by $\left(Y_{e}(x)-Y_{e}(y)\right) \mathscr{E}$, we can conclude that $\pi_{e}(u)=I_{\boldsymbol{K}_{e}}\left(=\right.$ the identity operator in $\left.K_{e}\right)$, i.e. $\pi_{e}(\cdot)$ is trivial if $d$ is of the form as in (5.0).

Suppose now that the function $b$ satisfying Theorem 5.2 arises from operator valued function, namely that $\mathscr{E}$ is a complex Hilbert space, $b_{x}(f, g)=(B(x) f, g)$, where $B(x) \in L(\mathscr{E})$ for $x$ in the group $X$. Then the canonical factorization for related $c_{x, y}^{e}$ function yields that $Y_{e}(x)$ are linear bounded operators from $\mathscr{E}$ into $K_{e}$ and

$$
Y_{e}(y)^{*} Y_{e}(x)=B\left(y^{-1} x\right)-B(x)-B\left(y^{-1}\right) .
$$

To the structural function there corresponds the operator function

$$
D\left(x, y ; x^{\prime}, y^{\prime}\right)=\left(Y_{e}\left(x^{\prime}\right)-Y_{e}\left(y^{\prime}\right)\right)^{*}\left(Y_{e}(x)-Y_{e}(y)\right)
$$

and for $u, v \in X$ we have

$$
D\left(u x, u y ; v x^{\prime}, v y^{\prime}\right)=\left(Y_{e}\left(x^{\prime}\right)-Y_{e}\left(y^{\prime}\right)\right)^{*} \pi_{e}\left(v^{-1} u\right)\left(Y_{e}(x)-Y_{e}(y)\right)
$$

which implies "orthodox" dilation type relations:

$$
\begin{aligned}
& D\left(u x, u ; v x^{\prime}, v\right)=Y_{e}\left(x^{\prime}\right)^{*} \pi_{e}\left(v^{-1} u\right) Y_{e}(x), \\
& D\left(u, u y ; v, v y^{\prime}\right)=Y_{e}\left(y^{\prime}\right)^{*} \pi_{e}\left(v^{-1} u\right) Y_{e}(y),
\end{aligned}
$$

simply because $Y_{e}(e)=0$.

Now we will consider the case where $X$ is a topological group with unit $e$ and $b_{x}(f, g)$ is conditionally positive definite, i.e. $\tilde{b}_{x, y}(f, g)=b_{y^{-1}}(f, g)$ is conditionally positive definite. It is usefull to define for a group $X$ the class $\mathrm{CP}_{0}(X)$ as the class of scalar valued functions $b_{x}(x \in X)$ such that the following conditions hold true:

(i) $(x, y) \rightarrow b_{y^{-1}}$ is conditionally positive definite;

(ii) $b_{x}=\overline{b_{x^{-1}}}$ (hermitian symmetry);

(iii) $b_{e}=0$ (normalization property).

If $b_{x} \in \mathrm{CP}_{0}(X)$ is continuous on $X$, then we say that $b_{x} \in \mathrm{CP}(X)$.

We will prove a slight extension of a lemma given in [17].

LEMMA 5.0. Let $X$ be a multiplicative group with unit $e$ and $b_{x}(x \in X)$ a function of class $\mathrm{CP}_{0}(X)$. Then

$$
\left|b_{y^{-1} x}-b_{x}-b_{y^{-1}}\right|^{2} \leqslant 4 \operatorname{Re} b_{x} \cdot \operatorname{Re} b_{y}
$$

for $x, y \in X$.

Proof. The function

$$
(x, y) \rightarrow b_{y^{-1}}-b_{x}-b_{y^{-1}}
$$

is positive definite because $b_{x}$ is of class $\mathrm{CP}_{0}(X)$. Indeed, by (i) and (iii) this follows from Proposition 4.0 by taking $x_{0}=e$. 
It follows then that

$$
\sum_{j, k \mid 1}^{2}\left(b_{x_{k}^{-1} x_{j}}-b_{x_{j}}-b_{x_{k}^{-1}}\right) \alpha_{j} \bar{a}_{k} \geqslant 0
$$

for $x_{1}, x_{2} \in X$ and $\alpha_{1}, \alpha_{2} \in C$. We take $x_{1}=x, x_{2}=y$ and $\alpha_{1}=s_{1}$ real and $a_{2}=s_{2} e^{i \theta}$ with real $s_{2}$ and $0 \leqslant \theta \leqslant 2 \pi$. It follows now that

$$
\left(-b_{x}-\bar{b}_{x}\right) s_{1}^{2}+\left(-b_{y}-\bar{b}_{y}\right) s_{2}^{2} \theta^{2 i \theta}+2 \operatorname{Re}\left[\left(b_{x^{-1} y}-b_{y}-b_{x}\right) s_{1} s_{2} e^{-i \theta}\right] \geqslant 0,
$$

that is,

$$
2 \operatorname{Re}\left[\left(b_{x^{-1} y}-b_{y}-b_{x}\right) s_{1} s_{2} e^{-i \theta}\right]-2 \operatorname{Re} b_{x} \cdot s_{1}^{2}-2 \operatorname{Re} b_{y} \cdot s_{2}^{2} \geqslant 0 .
$$

For suitable $\theta$

$$
b_{x^{-1} y}-b_{y}-b_{x^{-1}}=\left|b_{x^{-1} y}-b_{y}-b_{x^{-1}}\right| e^{i \theta}
$$

which by the previous inequality implies

$$
u\left(s_{1}, s_{2}\right)=\left|b_{x^{-1} y}-b_{y}-b_{x^{-1}}\right|^{2} s_{1} s_{2}-2 \operatorname{Re} b_{x} s_{1}^{2}-2 \operatorname{Re} b_{y} s_{2}^{2} \geqslant 0 .
$$

Since $s_{1}$ and $s_{2}$ are arbitrary reals the determinant related to the quadratic form $u\left(s_{1}, s_{2}\right)$ is non-negative which proves the claim.

The following lemma is now in order:

LEMMA 5.1. Suppose that $X$ is a topological group with unit $e$ and $b_{x}$ is of class $\mathrm{CP}_{0}(X)$. Then, if $b_{x}$ is continuous at $x=e$, then $b_{x}$ is continuous all over the group $X$, i.e. $b_{x}$ is of class $\mathrm{CP}(X)$.

Proof. $\left|b_{y x_{a}}-b_{y}\right| \leqslant\left|b_{x_{a}}\right|+2 \sqrt{\operatorname{Re} b_{x_{a}} \cdot \operatorname{Re} b_{y}} \rightarrow 0$ if $x_{a} \rightarrow e$.

The above two lemmas enable us to complete the Theorem 5.2 (with enclosed overthere notation) as follows:

THEOREM 5.3. Let $X$ be a topological group with unit e and let $b=\left\{b_{x}(f, g)\right\}$ satisfy the assumptions of Theorem 5.2. Suppose that $b_{e}(f, f)=0$ and $\overline{b_{x}(f, f)}=b_{x^{-1}}(f, f)$ for $x \in X, f \in \mathscr{E}$. Suppose that the function $x \rightarrow b_{x}(f, f)$ $i s$ continuous at $x=e$ for every $f \in \mathscr{E}$. Then the function $x \rightarrow Y_{e}(x) f$ is continuous on $X$ for every $f \in \mathscr{E}$ and $\pi_{e}(\cdot)$ is strongly continuous unitary representation of $X$.

Proof. Our assumptions imply that for every $f$ the function $x \rightarrow b_{x}(f, f)$ is of class $\mathrm{CP}_{0}(X)$. Lemma 5.1 yields that this function is continuous at every point of $X$. Hence for $x_{0} \in X, x \in X$

$$
\left\|Y_{e}\left(x_{0}\right) f-Y_{e}(x) f\right\|_{K_{e}}^{2}=-2 \operatorname{Re} c_{x-1}^{e} x_{0}(f, f) \rightarrow 0 .
$$

if $x \rightarrow x_{0} ; c^{e}, K_{e}, Y_{e}$ are associated at $e$ to $b$. Next, $\left(\pi_{e}(u) Y_{e}(x) f, Y_{e}(y) g\right)_{K_{e}}$ $=\left(\left(Y_{e}(u x)-Y_{e}(u)\right) f, Y_{e}(y) g\right)_{K_{e}}$. Keeping $x$ fixed we conclude by continuity of $Y_{e}(z) f$ that the map $u \rightarrow\left(\pi_{e}(u) h_{1}, h_{2}\right)$ is continuous in $u$ for $h_{1}, h_{2}$ 
which vary over a set which spans $K_{e}$. Since $\left\|\pi_{e}(u)\right\| \leqslant 1$ and $\pi(\cdot)$ is a unitary representation, we conclude therefore that $\pi_{e}(\cdot)$ is weakly, and consequently, a strongly continuous unitary representation of $X$.

6. Functions on the additive group of a Hilbert space. In this section we present a concrete model for applications of general theory developed in the previous sections. We deal with functions $b_{x}(f, g)$ where $x \in X$ and $X$ is a real separable Hilbert space. This leads us to dilation type Levy's Khintchine formula for suitable conditionally positive definite functions.

To begin with let us assume that $X$ is a real separable Hilbert space with inner product $\langle x, y\rangle$ and norm $|x|$, for $x, y \in X$.

The bounded linear operator $B$ in $X$ is called an $S$-operator if $B=B^{*}$, i.e. $B$ is symmetric and non-negative $-B \geqslant 0$, i.e. $\langle B x, x\rangle \geqslant 0$ for $x \in X$ and has a finite trace, i.e. the series $\sum_{n}\left\langle B e_{n}, e_{n}\right\rangle$ is convergent for some orthonormal basis $\left\{e_{n}\right\}$ of $X$, and consequently converges to the same sum for every orthonormal basis - we refer here to [16], [26].

Let $S_{X}$ be the totality of all $S$-operators in $X$. The totality of sets of the form $Q_{B}=\{x \in X ;\langle B x, x\rangle<1\}$ where $B \in S_{X}$ defines a system of neighbourhoods of zero. Translations of sets $Q_{B}$ by elements of $X$ define a topology on $X$ called the $S$-topology and $X$ with this topology becomes a linear topological space - see [26]; in particular:

\section{$X$ with S-topology is an additive topological group.}

It is plain that if a complex function $z(x)$ on $X$ is continuous in the $S$-topology at the poit $x_{0}$, then $z(x)$ is continuous at $x_{0}$ in norm topology of $X$, i.e. if $\left|x_{n}-x\right| \rightarrow 0$, then $z\left(x_{n}\right) \rightarrow z\left(x_{0}\right)$. If $\operatorname{dim} X=\infty$ then the norm $|\cdot|$ is not $\mathbb{S}$-continuous. If $\operatorname{dim} X<+\infty$, then $\mathbb{S}$-topology is equivalent with the norm topology.

Since now $X$ is considered as an additive topological group with S-topology. The function $b=\left\{b_{x}(f, g)\right\}(x \in X ; f, g \in \mathscr{E})$ is called of class $\mathrm{CP}(X ; \mathscr{E})$, if for every $f \in \mathscr{E}$ the scalar function $b_{x}(f, f)$ is of class $\mathrm{CP}(X)$, i.e. $b_{0}(f, f)=0,(x, y) \rightarrow b_{x-y}(f, f)$ is conditionally positive definite, i.e. $\tilde{b}$ is weakly conditionally positive definite, $\overline{b_{x}(f, f)}=b_{-x}(f, f)$ for $x \in X$ and $b_{x}(f, f)$ is $S$-continuous at $x=0$, and consequently, by Lemma 5.1, is continuous all over the space $X$. Our purpose is to give the description of functions of class $\mathrm{CP}(X ; \mathscr{E})$. It reduces to classical formulae if $\mathscr{E}=C^{\mathbf{l}}$, however there appear some new effects in case where $\operatorname{dim} \mathscr{E}>1$.

If $b_{x}$ is of class CP $(X)$, where $X$ is treated as an additive group with $S$-topology, then by (4.0) for every $\tau>0$ :

(i) The function $\varphi_{\tau}(x)=e^{\tau b_{x}}$ is positive definite on $X$ (i.e. $(x, y)$ $\rightarrow \varphi_{\tau}(x-y)$ is positive definite),

(ii) $\varphi_{\tau}(0)=1$, 
(iii) $\varphi_{\tau}(\cdot)$ is continuous in the S-topology of $X$, and consequently,

(iv) $\varphi_{\tau}(\cdot)$ is continuous in norm topology of $X$.

It follows then by (i)-(iii) from the Minlos-Sazonov theorem - see $[16],[6],[26]$ - that $\varphi_{\tau}(\cdot)$ is a Fourier transform of the unique probability measure $\mu_{\tau}$ on the $\sigma$-field $B(X)$ of Borel subsets of $X$, that is

$$
e^{\tau b}=\varphi_{x}(x)=\int_{X} e^{i\langle x, y\rangle} d \mu_{\tau}(y) \quad \text { for } x \in X .
$$

When taking $\tau=1 / n, n=1,2, \ldots$ we conclude that $\mu_{1}$ is a probability measure of an infinitely divisible random variable. It follows then from the Varadhan extension of Levy's-Khintchine formula to Hilbert spaces. (see [6], [16], [26] and [34]) that

$$
e^{b} x=e^{i\langle a, x\rangle-\langle B x, x\rangle+\int_{X} h(x, y) d \mu(u)}, \quad x \in X,
$$

where $a \in X, B$ is an $S$-operator, $\mu$ is a finite positive measure on $B(X)$ : vanishing on the singleton $\{0\}$ and

$$
h(x, y)=\left(e^{i\langle x, y\rangle}-1-\frac{i\langle x, y\rangle}{1+|y|^{2}}\right) \frac{1+|y|^{2}}{|y|^{2}}
$$

for $y \neq 0$. Notice now that

$$
h(x, y)=\left(e^{i\langle x, y\rangle}-1\right)+\frac{e^{i\langle x, y\rangle}-1-i\langle x, y\rangle}{|y|^{2}}
$$

for $y \neq 0$, which by the general inequality

$$
\left|e^{i s}-1-i s\right| \leqslant s^{2} / 2 \quad(s \text { real })
$$

proves that for every fixed $x$ the function $h(x, y)$ is bounded as the function of $y \neq 0$. Indeed, (6.2) implies that

$$
|h(x, y)| \leqslant 2+|x|^{2} / 2 \quad \text { for } x \in X, y \neq 0 .
$$

Hence, if $x_{n} \rightarrow x$ in norm, then obviously $h\left(x_{n}, y\right) \rightarrow h_{n}(x, y)$ for $y \neq 0$ and by

$$
\sup _{\boldsymbol{y} \neq 0, n}\left|h\left(x_{n}, y\right)\right|<+\infty
$$

which by dominated convergence, since $\mu_{1}(\{0\})=0$, proves that $\int_{X} h\left(x_{n}, y\right) d \mu(y) \rightarrow \int_{X} h(x, y) d \mu(y)$. Since $y \in X$ and $B$ is continuous, we conclude that the exponent on the right-hand side of (6.1) is continuous in norm. Since $b_{x}$ does the same and both are zero at $x=0$ and $X$ is a connected space, we conclude that

$$
b_{x}=i\langle a, x\rangle-\langle B x, x\rangle+\int_{\boldsymbol{X}} h(x, y) d \mu(y) \quad \text { for } x \in X
$$


where $a \in X, B$ is an $S$-operator and $\mu$ a positive finite measure on $B(X)$ vanishing at the point $y=0$. All we said means that every function of class $\mathrm{CP}(X)$ is of form (6.4). This is in fact equivalent to Varadhan extension of classical Levy-Khintchine result. Since this result was already proved, we found it convenient although a little bit artificial, to use it for getting (6.4) just for the sake of brevity. The uniqueness properties of parameters $a, B, \mu$ appearing in (6.4) are included in the lemma below, which is crucial for our purposes. The idea of the proof of this lemma is essentially due to Gichman-Skorohod [6]; see also [16].

LEMMA 6.0. Let $X$ be a real separable Hilbert space. Suppose that we are given the functions $u_{1}, u_{2}: X \rightarrow C$ such that

$$
u_{i}(t(x+y))=t u_{i}(x)+t u_{i}(y) \quad \text { for } t \in \boldsymbol{R}, x, y \in X, i=1,2,
$$

and functions $v_{1}, v_{2}: X \times X \rightarrow C$ such that

$$
\begin{aligned}
& v_{i}(x, y) \text { is real linear in } x \text { and } y, \text { i.e. } \\
& v_{i}(t(x+z), y)=t v_{i}(x, y)+t v_{i}(z, y), \\
& v_{i}(x, t(y+z))=t v_{i}(x, y)+t v_{i}(x, z) \\
& \qquad \text { for } x, y \in X, t \in \boldsymbol{R} \text { and } i=1,2 .
\end{aligned}
$$

Let $\mu_{1}, \mu_{2}$ be two complex measures on $B(X)$ such that

$$
\mu_{1}(\{0\})=0=\mu_{2}(\{0\}) .
$$

Then, if

$$
\begin{aligned}
& u_{1}(x)+v_{1}(x, x)+\int_{X}\left(e^{i\langle x, y\rangle}-1-\frac{i\langle x, y\rangle}{1+|y|^{2}}\right) \frac{1+|y|^{2}}{|y|^{2}} d \mu_{1}(y) \\
& =u_{2}(x)+v_{2}(x, x)+\int_{X}\left(e^{i\langle x, y\rangle}-1-\frac{i\langle x, y\rangle}{1+|y|^{2}}\right) \frac{1+|y|^{2}}{|y|^{2}} d \mu_{2}(y)
\end{aligned}
$$

for all $x \in X$, then $u_{1}(x)=u_{2}(x), v_{1}(x, x)=v_{2}(x, x)$ for all $x \in X$ and $\mu_{1}$ $=\mu_{2}$.

Proof. Let us write for $x \in X, y \in X$

$$
\begin{aligned}
& h(x, y)=\left(e^{i\langle x, y\rangle}-1-\frac{i\langle x, y\rangle}{1+|y|^{2}}\right) \frac{1+|y|^{2}}{|y|^{2}} \quad \text { if } y \neq 0, \\
& h(x, 0)=0 \quad \text { for all } x .
\end{aligned}
$$

Since $\mu_{1}, \mu_{2}$ vanish on the singleton $\{0\}$, we get from $(6.5)-(6.8)$ that for every real $t$ and $x \in X$

$$
\begin{aligned}
t u_{1}(x)+t^{2} v_{1}(x, x)+\int_{X} h(t x, y) d \mu_{1}(y) & \\
& =t u_{2}(x)+t^{2} v_{2}(x, x)+\int_{x} h(t x, y) d \mu_{2}(y)
\end{aligned}
$$


and consequently for $t>0, x \in X$

$$
\begin{aligned}
\frac{u_{1}(x)}{t}+v_{1}(x, x)+\int_{X} & \frac{h(t x, y)}{t^{2}} d \mu_{1}(y) \\
& =\frac{u_{2}(x)}{t}+v_{2}(x, x)+\int_{\Sigma} \frac{h(t x, y)}{t^{2}} d \mu_{2}(y) .
\end{aligned}
$$

Inequality (6.2) yields that for $x \in X, y \neq 0$

$$
\frac{|h(t x, y)|}{t^{2}} \leqslant \frac{2}{t^{2}}+\left|\frac{e^{i t\langle x, y\rangle}-1-i t\langle x, y\rangle}{t^{2}|y|^{2}}\right| \leqslant \frac{2}{t^{2}}+\frac{|x|^{2}}{2}
$$

Since

$$
\frac{e^{i t\langle x, y\rangle}-1}{t^{2}|y|^{2}}-\frac{i\langle x, y\rangle}{t|y|^{2}} \underset{t \rightarrow+\infty}{\rightarrow} 0 \quad(x \in X, y \neq 0),
$$

we conclude therefore by dominated convergence theorem that, since $\mu_{1}, \mu_{2}$ vanish on $\{0\}$,

$$
\lim _{t \rightarrow \infty} \int_{\boldsymbol{X}} \frac{h(t x, y)}{t^{2}} d \mu_{1}(y)=0=\lim _{t \rightarrow \infty} \int_{X} \frac{h(t x, y)}{t^{2}} d \mu_{2}(y)
$$

which by $(6.9)$ shows that $v_{1}(x, x)=v_{2}(x, x)$ for $x \in X$.

Let $z_{1}(x)$ be the left-hand side of $(6.8)$ and $z_{2}(x)$ the right-hand one. Suppose $\left\{e_{k}\right\}$ is an orthonormal basis of $X$. We have for real $t$ that for $x \in X, p=1,2$

$$
\begin{gathered}
z_{p}\left(x+t e_{k}\right)=u_{p}(x)+t u_{p}\left(e_{k}\right)+v_{p}(x, x)+t v_{p}\left(e_{k}, x\right)+t v_{p}\left(x, e_{k}\right)+ \\
+t^{2} v_{p}\left(e_{k}, e_{k}\right)+\int_{X}\left(e^{i\langle x, y\rangle} e^{i\left\langle\left\langle e_{k}, y\right\rangle\right.}-1-\frac{i\langle x, y\rangle}{1+|y|^{2}}-\frac{i t\left\langle e_{k}, y\right\rangle}{1+|y|^{2}}\right) \frac{1+|y|^{2}}{|y|^{2}} d \mu_{p}(y)
\end{gathered}
$$

and

$$
\begin{aligned}
& z_{p}\left(x-t e_{k}\right) \\
& =u_{p}(x)-t u_{p}\left(e_{k}\right)+v_{p}(x, x)-t v_{p}\left(x, e_{k}\right)-t v_{p}\left(e_{k}, x\right)+t^{2} v_{p}\left(e_{k}, e_{k}\right)+ \\
& \quad+\int_{x}\left(e^{i\langle x, y\rangle} e^{-i t\left\langle e_{k}, y\right\rangle}-1-\frac{i\langle x, y\rangle}{1+|y|^{2}}+\frac{i t\left\langle e_{k}, y\right\rangle}{1+|y|^{2}}\right) \frac{1+|y|^{2}}{|y|^{2}} d \mu_{p}(y)
\end{aligned}
$$

We derive from the above equalities that for $x \in X$, real $t$ and any $e_{k}$

$$
\begin{aligned}
\frac{1}{2}\left[z_{p}\left(x+t e_{k}\right)+\right. & \left.z_{p}\left(x-t e_{k}\right)\right]=u_{p}(x)+v_{p}(x, x)+t^{2} v_{p}\left(e_{k}, e_{k}\right)+ \\
& +\int_{X}\left(e^{i\langle x, y\rangle} \cdot \cos t\left\langle y, e_{k}\right\rangle-1-\frac{i\langle x, y\rangle}{1+|y|^{2}}\right) \frac{1+|y|^{2}}{|y|^{2}} d \mu_{p}(y) .
\end{aligned}
$$

14 - Annales Polontei Mathematicl XIII 


\section{Consequently}

$$
\begin{aligned}
z_{p}(x)-\frac{1}{2}\left(z_{p}(x+\right. & \left.\left.t e_{k}\right)+z_{p}\left(x-t e_{k}\right)\right) \\
= & \int_{x} e^{i\langle x, y\rangle}\left(1-\cos t\left\langle y, e_{k}\right\rangle\right) \frac{1+|y|^{2}}{|y|^{2}} d \mu_{p}(y)-t^{2} v_{p}\left(e_{k}, e_{k}\right) .
\end{aligned}
$$

Since $z_{1}(x)=z_{2}(x)$ for all $x \in X$ and $v_{1}\left(e_{k}, e_{k}\right)=v_{2}\left(e_{k}, e_{k}\right)$, we conclude that for $x \in X, t \in \boldsymbol{R}$ and every $e_{k}$

$$
\begin{aligned}
\int_{X} e^{i\langle x, y\rangle}\left(1-\cos t\left\langle y, e_{k}\right\rangle\right) & \frac{1+|y|^{2}}{|y|^{2}} d \mu_{1}(y) \\
& =\int_{X} e^{i\langle x, y\rangle}\left(1-\cos t\left\langle y, e_{k}\right\rangle\right) \frac{1+|y|^{2}}{|y|^{2}} d \mu_{2}(y) .
\end{aligned}
$$

We take now $c_{k}>0$ such that $\sum_{k} c_{k}<+\infty$ and just conclude that the series $\sum_{k} c_{k}\left(1-\cos t\left\langle y, e_{k}\right\rangle\right)$ converges uniformly in $t$ and $y$. It follows then from $(6.10)$ that

$$
\begin{aligned}
\int_{X} e^{i\langle x, y\rangle} \sum_{k} c_{k}(1- & \left.\cos t\left\langle y, e_{k}\right\rangle\right) \frac{1+|y|^{2}}{|y|^{2}} d \mu_{1}(y) \\
= & \int_{X} e^{i\langle x, y\rangle} \sum_{k} c_{k}\left(1-\cos t\left\langle y, e_{k}\right\rangle\right) \frac{1+|y|^{2}}{|y|^{2}} d \mu_{2}(y),
\end{aligned}
$$

which, by integrating with respect to $t$ over the interval $[-a, a] .(a>0)$ gives by Fubini theorem that for $x \in X$

$$
\int_{X} e^{i\langle x, y\rangle} s(y) d \mu_{1}(y)=\int_{X} e^{i\langle x, y\rangle} s(y) d \mu_{2}(y),
$$

where

$$
\begin{aligned}
s(y) & =\sum_{k} c_{k}\left(1-\frac{\sin a\left\langle y, e_{k}\right\rangle}{a\left\langle y, e_{k}\right\rangle}\right) \quad\left(\text { we put } \frac{\sin 0}{0}=1\right. \text { by definition) } \\
& =\int_{-a}^{+a}\left[\sum_{k} c_{k}\left(1-\cos t\left\langle y, e_{k}\right\rangle\right)\right] d t .
\end{aligned}
$$

If $y \neq 0$, then for some $e_{k}\left\langle y, e_{k}\right\rangle \neq 0$. Since $1-(\sin u) / u>0$ for all $u \neq 0$, we conclude that $s(y)>0$ if $y \neq 0$. Since the Fourier transform over $X$ determines the measure in the unique way (see [16], 7.6.3) and $\mu_{1}, \mu_{2}$ vanish on the singleton $\{0\}$, the measures $s(y) d \mu_{1}, s(y) d \mu_{2}$ are equal by (6.11), which, since $y \neq 0$ implies $s(y)>0$, proves that $\mu_{1}=\mu_{2}$. We proved already that $v_{1}=v_{2}$. It follows now that $u_{1}=u_{2}$ which completes the proof of the lemma. 
For the sake of brevity the function $v(x, y)=\sum_{j \mid 1}^{4} a_{j}\left\langle B_{j} x, y\right\rangle$, where $a_{j} \in C$ and $B_{j}$ are $S$-operators will be called a nuclear bilinear form. It is plain that $v$ is symmetric, i.e. $v(x, y)=v(y, x)$.

For the complex linear space $\mathscr{E}$ and the Hilbert real space $X$ the scalar function $u(x ; f, g)$ bilinear in $f, g$, and real linear in $X$ will be called an elementary form.

We are ready to prove our basic theorem, namely

THEOREM 6.0. Let $X$ be a real separable Hilbert space and $b .=\left\{b_{x}(f, g)\right\}$ $(f, g \in \mathscr{E})$ the function of class $\mathrm{CP}(X ; \mathscr{E})$. Then there are the unique nuclear forms $v_{f, g}$, the unique elementary forms $u_{f, g}$ and the unique family $\mu_{f, o}$ $(f, g \in \mathscr{E})$ of measures on $B(X)$, all vanishing on $\{0\}$, such that for $x \in X$

$$
\begin{aligned}
b_{x}(f, g)=u_{f, g}(x)+ & v_{f, g}(x, x)+ \\
& +\int_{\mathbf{x}}\left(e^{i\langle x, y\rangle}-1-\frac{i\langle x, y\rangle}{1+|y|^{2}}\right) \frac{1+|y|^{2}}{|y|^{2}} d \mu_{f, g}(y),
\end{aligned}
$$

and $u_{f, g}(x), v_{f, g}(x, x)$ are bilinear in $f, g \in \mathscr{E}, \mu_{f, g}(\sigma)$ is bilinear in $f, g \in \mathscr{E}$ for every $\sigma \in B(X)$ and $\mu_{f, f}(\sigma) \geqslant 0$ for $f \in \mathscr{E}, \sigma \in B(X)$.

Proof. The uniqueness statements follow from Lemma 6.0 and the fact that $b_{x}(f, g)$ is bilinear in $f, g \in \mathscr{E}$. The proof of existence part of assertion runs as follows: the already proved uniqueness implies that $a, B$ and $\mu$ in (6.4) are unique for $b_{x}$ of class $\operatorname{CP}(X)$. Hence for every $f \in \mathscr{E}$ we have the unique representation

$$
b_{x}(f, f)=i\langle a(f), x\rangle-\langle B(f) x, x\rangle+\int_{x} h(x, y) d \mu_{f}(y),
$$

where $a(f) \in X, B(f)$ is an $S$-operator, $h(x, y)$ is defined as in the proof of Lemma 6.0 and $\mu_{f}$ is a finite positive measure vanishing on $\{0\}$.

We define for $x, y \in X, f, g \in \mathscr{E}$

$$
\begin{aligned}
& u_{f, g}(x)=\frac{1}{4} i[\langle a(f+g), x\rangle-\langle a(f-g), x\rangle+i\langle a(f+i g), x\rangle- \\
& -i\langle a(f-i f), x\rangle], \\
& v_{f, g}(x, y)=-\frac{1}{4}[\langle B(f+g) x, y\rangle-\langle B(f-g) x, y\rangle+i\langle B(f+i g) x, y\rangle- \\
& -i\langle B(f-i g) x, y\rangle] \\
& \mu_{f, \sigma}=\frac{1}{4}\left[\mu_{f+g}-\mu_{f-g}+i \mu_{f+i g}-i \mu_{f-i g}\right] .
\end{aligned}
$$

The polarization formula for $b_{x}(f, g)$ yields that (6.12) holds true. Using now Lemma 6.0 and having in view that $b_{x}(f, g)$ is bilinear in $f, g$, we see that $u_{f, g}, v_{f, g}, \mu_{f, g}$ are bilinear in $f, g$, and $\mu_{f}=\mu_{f, f}-$ hence $\mu_{f, f}(\sigma) \geqslant 0$ for $\sigma \in B(X)$. It is plain that $u_{f, g}$ are elementary forms and $v_{f, g}$ nuclear ones, which completes the proof. 
COROLlARY 6.0. The forms $v_{f, \theta}(x, y)$ are symmetric, i.e. $v_{f, \theta}(x, y)$ $=v_{f, g}(y, x)$ and consequently are determined in the unique way by related quadratic forms $\hat{v}_{f, g}(x)=v_{f, g}(x, x)$. Moreover, $u_{f, g}(x), v_{f, g}(x, y)$ are norm continuous in $x$ and $y$.

Following the classical terminology we write $v_{f, g}(x, y)=-\frac{1}{2} G(f, g$; $x, y$ ) and call $G$ the Gaussian part of $b$. We write

$$
\boldsymbol{P}(f, g ; x)=\int_{\boldsymbol{X}} h(x, y) d \mu_{f, g}(y)
$$

and call $P(-)$ the Poisson part of $b$. The elementary part $u_{f, g}(x)$ is conditionally positive definite on the group $X$. Indeed, if $\sum_{j \mid 1}^{n} f_{j}=0\left(f_{j} \in \mathscr{E}\right)$,
then for $x_{1}, \ldots, x_{n} \in X$

$$
\sum_{j, k \mid 1}^{n} u_{f_{j}, f_{k}}\left(x_{j}-x_{k}\right)=\sum_{k \mid 1}^{n} u_{j \mid 1}^{n} f_{j}, f_{k}\left(x_{k}\right)-\sum_{j \mid 1}^{n} u \sum_{k \mid 1}^{n} f_{k}, f_{j}\left(x_{j}\right)=\mathbf{0} .
$$

Notice now that in our theorem we assumed merely that $b$ is of class $\operatorname{CP}(X ; \mathscr{E})$ which among others means that the function $b=\left\{b_{x}(f, g)\right\}$ is weakly conditionally positive definite, i.e. for every $f \in \mathscr{E}$ the scalar function $(x, y) \rightarrow b_{x-y}(f, f)$ is conditionally positive definite. Our next basic result says that for Poisson type functions this weak conditional positive definiteness implies the conditional positive definiteness.

THEOREM 6.1. The Poisson part of the function $b=\left\{b_{x}(f, g)\right\}$ of class $\mathrm{CP}(X ; \mathscr{E})$ is conditionally positive definite.

Proof. We will apply some essentially dilation type arguments. Recall first that the measures $\mu_{f, g}$ of Theorem 6.0 are bilinear in $f, g$ and $\mu_{f, f}(\sigma) \geqslant 0$ for $\sigma \in B(X)$. It follows then from Proposition 2.1 that there are a complex Hilbert space $K$, a linear map $R: \mathscr{E} \rightarrow K$ and the spectral measure $E(\sigma)$ in $K(\sigma \in B(X))$ such that $\mu_{f, g}(\sigma)=(E(\sigma) R f, R g)_{K}$. It follows that

$$
P(f, g ; x)=\int_{X} h(x, y) d\left(E_{y} R f, R g\right)_{K} \cdot
$$

Notice that if $K$ is minimal, and in all what follows we assume that this is the case, then $E(\{0\})=0$, because $\mu_{f, \theta}$ vanish on $\{0\}$.

This is an elementary exercise to check that for every fixed $y \in X$ the function

$$
(x, z) \rightarrow h(x-z, y)
$$

is conditionally positive definite and hermitian symmetric, i.e. $\overline{h(x-z, y)}$ $=h(z-x, y)$. It follows that the function

$$
\cdot(x, z) \rightarrow e^{\tau h\left(x-z_{\bullet}, v\right)}
$$


is positive definite for every $\tau>0, y$ being fixed. Having in view Example 4.0 , it is enough for our purpose to prove that the operator function

$$
\Phi_{\tau}(x-y)=\int_{X} e^{\tau h(x-y, z)} d E_{\varepsilon}
$$

(spectral integral) is positive definite, i.e. for $k^{\prime}, k^{\prime \prime} \in K\left(\Phi_{\tau}(\cdot) k^{\prime}, k^{\prime \prime}\right)_{K}$ $>>0$ for every $\tau>0$. Indeed, suppose just that $h_{1}, \ldots, h_{n} \in K$ and $x_{1}, \ldots$ $\ldots, x_{n} \in X$. Then

$$
\sum_{j, k \mid 1}^{n}\left(\Phi_{\tau}\left(x_{j}-x_{k}\right) h_{j}, h_{k}\right)_{K}=\sum_{j, k \mid \mathrm{I}}^{n}\left(e^{\chi^{\tau} \tau h\left(x_{j}-x_{k}, v\right)} d\left(E_{y} h_{j}, h_{k}\right)_{K},\right.
$$

because $\Phi_{\tau}(x-y)=e^{\int^{\tau} \tau h(x-y, z)} d E_{z}$ by von Neumann-Stone operational calculus.

Since we have only a finite number of $x$-es, for every $\varepsilon>0$ there is a partition $\left\{\sigma_{m}\right\}$ of $X, \sigma_{m} \in B(X)$ such that for the fixed $\tau>0$ and $j, k=1, \ldots$ $\ldots, n$

$$
\left\|\int_{\boldsymbol{X}} e^{\tau \hbar\left(x_{j}-x_{k}, y\right)} d E_{y}-\sum_{m} e^{\tau \hbar\left(x_{j}-x_{k}, y_{m}\right)} E\left(\sigma_{m}\right)\right\|_{\underline{K}}<\varepsilon
$$

where $y_{m} \in \sigma_{m}$ and \|\|$_{K}$ stands for the operator norm in $K$. It follows that it suffices to show that

Since

$$
a=\sum_{j, k} \sum_{m} e^{\tau h\left(x_{j}-x_{k}, y_{m}\right)}\left(E\left(\sigma_{m}\right) h_{j}, h_{k}\right)_{K} \geqslant 0 .
$$

$$
a=\sum_{m} \delta_{m} \quad \text { where } \quad \delta_{m}=\sum_{j, k \mid 1}^{n} e^{\tau h\left(x_{j}-x_{l}, y_{m}\right)}\left(E\left(\sigma_{m}\right) h_{j}, E\left(\sigma_{m}\right) h_{k}\right)_{K},
$$

all we need to prove is that $\delta_{m} \geqslant 0$ for all $m$. Now, since the matrix $\left\{e^{\tau h\left(x_{j}-x_{k}, y_{m}\right)}\right\}_{j, k=1, \ldots, n}$ is positive definite (because the function of (6.13) is positive definite for every $y)$ and the matrix $\left\{\left(E\left(\sigma_{m}\right) h_{j}, E\left(\sigma_{m}\right) h_{k}\right)\right\}$ is also positive definite, the Schur's lemma yields that $\delta_{m} \geqslant 0$.

The Gaussian part of a function of class $\operatorname{CP}(X ; \mathscr{E})$ need not be definite if $\operatorname{dim} X \geqslant 2$. The example below is just the suitable reinterpretation of an example due to Choi - see [21], Example 6.0. Let $\mathscr{E}$ be the algebra of all complex $2 \times 2$ matrices with usual algebraic operations. We take $X=\boldsymbol{R}^{2} \cdot f^{*}$ stands for the hermitian adjoint of $f \in \mathscr{E}$ and $e=\left\{\delta_{j k}\right\}_{j, k=1,2}$. For $g \in \mathscr{E}$ we define $s(g)=$ trace of $g$ and define

$$
\varphi(f)=s(f) e-f .
$$

For $x \in \boldsymbol{R}^{2}$ and $f, g \in \mathscr{E}$ we write

$$
b_{x}(f, g) \stackrel{\text { af }}{=}-\left(\varphi\left(g^{*} f\right) x, x\right) \quad\left((\cdot, \cdot) \text { - the inner product in } C^{2}\right) .
$$


$\varphi\left(f^{*} f\right)=s\left(f^{*} f\right) e-f^{*} f$ is a positive definite symmetric matrix. Assuming $\sum_{j \mid 1}^{n} \alpha_{j}=0\left(a_{j} \in C\right)$ and fixing $f \in \mathscr{E}$, we have

$$
\begin{aligned}
a= & \sum_{j, k \mid 1}^{n} b_{x_{j}-x_{k}}(f, f) a_{j} \bar{a}_{k}=-\sum_{j, k \mid 1}^{n}\left(\varphi\left(f^{*} f\right)\left(x_{j}-x_{k}\right),\left(x_{j}-x_{k}\right)\right) a_{j} \bar{a}_{k} \\
= & -\sum_{k \mid 1}^{n} \sum_{j \mid 1}^{n}\left[\left(\varphi\left(f^{*} f\right) x_{j}, x_{j}\right) a_{j}\right] \bar{a}_{k}-\sum_{j \mid 1}^{n} \sum_{k \mid 1}^{n}\left[\left(\varphi\left(f^{*} f\right) x_{k}, x_{k}\right) \bar{a}_{k}\right] a_{j}+ \\
& +\sum_{j, k \mid 1}^{n}\left(\varphi\left(f^{*} f\right) x_{j}, x_{k}\right) a_{j} \bar{a}_{k}+\sum_{j, k \mid 1}^{n}\left(\varphi\left(f^{*} f\right) x_{k}, x_{j}\right) a_{j} \bar{a}_{k}
\end{aligned}
$$

which since $\sum_{j \mid \mathbf{1}}^{n} a_{j}=\sum_{k \mid \mathbf{1}}^{n} \bar{a}_{k}=0$ implies that

$$
a=\left(\varphi\left(f^{*} f\right) \sum_{j \mid 1}^{n} \alpha_{j} x_{j}, \sum_{k \mid 1}^{n} \alpha_{k} x_{k}\right)+\left(\varphi\left(f^{*} f\right) \sum_{k \mid 1}^{n} \bar{d}_{k} x_{k}, \sum_{j \mid 1}^{n} \bar{a}_{j} x_{j}\right) \geqslant 0 .
$$

Obviously, $b_{0}(f, f)=0$ and $\overline{b_{x}(f, f)}=\overline{-\left(\varphi\left(f^{*} f\right) x, x\right)}=-\left(\varphi\left(f^{*} f\right) x, x\right)$ $=b_{-x}(f, f)=-\langle B(f) x, x\rangle_{\mathbf{R}^{2}}$, because the matrix $\varphi\left(f^{*} f\right)$ is hermitian symmetric. Plainly, $b_{x}(f, f)$ is continuous in $x$. Hence $b_{x}(f, g)$ is of class $\mathbf{C P}\left(\boldsymbol{R}^{2} ; \mathscr{E}\right)$ and reduces to its Gaussian part.

Our claim is that $b$ is not conditionally positive definite. Suppose in contrary that $b_{x}(f, g)$ is conditionally positive definite. This implies, by Proposition 4.0 by taking $x_{0}=0$, that the function

$$
c_{x, y}(f, g)=b_{x-y}(f, g)-b_{x}(f, g)-b_{y}(f, g) \quad\left(b_{-y}(f, g)=b_{y}(f, g)\right)
$$

is positive definite. Hence if $f_{1}, \ldots, f_{4} \in \mathscr{E}$ and $x_{1}, \ldots, x_{4} \in \boldsymbol{R}^{2}$ we must have

$$
b=\sum_{j, k \mid 1}^{4}\left(b_{x_{j}-x_{k}}\left(f_{j}, f_{k}\right)-b_{x_{j}}\left(f_{j}, f_{k}\right)-b_{x_{k}}\left(f_{j}, f_{k}\right)\right) \geqslant 0
$$

i.e.

$$
\begin{aligned}
b & =\sum_{j, k \mid 1}^{4}\left[\left(\varphi\left(f_{k}^{*} f_{j}\right) x_{j}, x_{j}\right)+\left(\varphi\left(f_{k}^{*} f_{j}\right) x_{k}, x_{k}\right)-\left(\varphi\left(f_{k}^{*} f_{j}\right)\left(x_{j}-x_{k}\right),\left(x_{j}-x_{k}\right)\right)\right] \\
& =\sum_{j, k \mid 1}^{4}\left[\left(\varphi\left(f_{k}^{*} f_{j}\right) x_{j}, x_{k}\right)+\left(\varphi\left(f_{k}^{*} f_{j}\right) x_{k}, x_{j}\right)\right] \geqslant 0 .
\end{aligned}
$$

We take

$$
f_{1}=\left(\begin{array}{ll}
1 & 0 \\
0 & 0
\end{array}\right), \quad f_{2}=\left(\begin{array}{ll}
0 & 1 \\
0 & 0
\end{array}\right), \quad f_{3}=\left(\begin{array}{ll}
0 & 0 \\
0 & 1
\end{array}\right), \quad f_{4}=\left(\begin{array}{ll}
0 & 0 \\
1 & 0
\end{array}\right)
$$


and $x_{1}=(1,0), x_{2}=(0,1), x_{3}=(0,1), x_{4}=(1,0)$. It. is easy to eheck that $s\left(f_{k}^{*} f_{j}\right)=\delta_{j k}$ and

$$
\sum_{j, k \mid 1}^{4}\left(\varphi\left(f_{k}^{*} f_{j}\right) x_{j}, x_{k}\right)=\sum_{j, k \mid 1}^{4} s\left(f_{k}^{*} f_{j}\right)\left(x_{j}, x_{k}\right)-\left\|\sum_{i \mid 1}^{4} f_{i} x_{i}\right\|^{2}=4-8=-4,
$$

because $\sum_{i \mid 1}^{4} f_{i} x_{i}=(2,2)$. On the other hand, by direct computation $\sum_{j, k \mid 1}^{4}\left(f_{k} x_{j}, f_{j} x_{k}\right)=4$ which implies that

$$
\sum_{j, k \mid 1}^{4}\left(\varphi\left(f_{k}^{*} f_{j}\right) x_{k}, x_{j}\right)=\sum_{j, k \mid 1}^{4} \delta_{j k}\left(x_{k}, x_{j}\right)-4=0 .
$$

Consequently, summing up we get that in this case $b=-4$. This is the desired contradiction with the assumption that $b_{x}(f, g)$ is conditionally positive definite and just completes the proof of our claim.

The following theorem appears now as natural:

THEOREM 6.2. The function $b$ of class $\mathrm{CP}(X ; \mathscr{E})$ is conditionally positive definite if and only if the Gaussian part of $b$ is conditionally positive definite.

Proof. If the Gaussian part of $b$ is conditionally positive definite, then, since the elementary part of $b$ as well the Poisson part of $b$ (by Theorem 6.1) share this property and then consequently $b$ is conditionally positive definite.

Suppose that $b$ is conditionally positive definite and let

$$
b_{x}(f, g)=i u((f, g), x)-\frac{1}{2} G(f, g ; x, x)+P(f, g ; x)
$$

be its canonical Levy's-Khintchine representation of Theorem 6.0. For real $t>0$ we have

$$
b_{t x}(f, g)=i t u(f, g ; x)-\frac{1}{2} t^{2} G(f, g ; x, x)+P(f, g ; t x)
$$

which implies that

$$
\frac{b_{t x}(f, g)}{t^{2}}=\frac{i u(f, g ; x)}{t}-\frac{1}{2} G(f, g ; x, x)+\frac{P(f, g ; t x)}{t^{2}} .
$$

Since the first and the last part of the above equality tend to zero if $t \rightarrow \infty$ (see the proof of Lemma 6.0), $-\frac{1}{2} G(f, g ; x, x)$ as a limit of conditionally positive definite functions $b_{x}^{\ell}(f, g)=b_{t x}(f, g) / t^{2}$ for $t \rightarrow \infty$ is conditionally positive definite.

The above theorem is interesting only if $\operatorname{dim} X \geqslant 2$, because of the following:

THEOREM 6.3. Every function of class $\operatorname{CP}\left(\boldsymbol{R}^{\mathbf{1}} ; \mathscr{E}\right)$ (E्E arbitrary) is conditionally positive definite. 
Proof. Having in view Theorem 6.2, it is sufficient to prove that Gaussian function $b_{x}(f, g), x \in \boldsymbol{R}^{1}$, is conditionally positive definite. Suppose just that $b$ is Gaussian. Then $b_{x}(f, g)=-\frac{1}{2} G(f, g ; x, x)$, where $G(f, g ; x, y)$ is bilinear in $f, g$ and real bilinear in $x, y$. Assume that $\sum_{j \mid 1}^{n} f_{j}$
$=0$. Then

$$
\begin{aligned}
2 \sum_{j, k \mid 1}^{n} b_{x_{j}-x_{k}}\left(f_{j}, f_{k}\right) & =-\sum_{j, k \mid 1}^{n} G\left(f_{j}, f_{k} ; 1,1\right)\left(x_{j}-x_{k}\right)^{2} \\
& =G\left(\sum_{j \mid 1}^{n} x_{j} f_{j}, \sum_{k \mid 1}^{n} x_{k} f_{k} ; 1,1\right) \geqslant 0,
\end{aligned}
$$

because $-b_{x}(h, h)=G(h, h ; x, x)=\langle B(h) x, x\rangle_{\boldsymbol{R}^{1}}=B(h)|x|^{2}$ for $h \in \mathscr{E}$; in this case where $B(h) \geqslant 0$ (see the proof of Theorem 6.0).

The canonical decomposition (6.12) of function $b_{x}(f, g)$ of the above theorem can we rewritten in the form

$$
b_{x}(f, g)=i u(f, g) x+\int_{-\infty}^{+\infty}\left(e^{i x y}-1-\frac{i x y}{1+y^{2}}\right) \frac{1+y^{2}}{y^{2}} d \mu_{f, g}(y)
$$

where the value of the function

$$
h(x, y)=\left(e^{i x y}-1-\frac{i x y}{1+y^{2}}\right) \frac{1+y^{2}}{y} \quad(y \neq 0)
$$

at $y=0$ is understood as

$$
h(x, 0) \stackrel{\text { df }}{=} \lim _{\substack{y \rightarrow 0 \\ y \neq 0}} h(x, y)=-\frac{x^{2}}{2} \quad \text { and } \quad \mu_{f, g}(\{0\})=G(f, g) .
$$

So, $h(x, y)$ defined for $x, y \neq 0$ extends to a function continuous for all $x, y \in \boldsymbol{R}^{1} ; u(\cdot, \cdot)$ and $\mu_{f, g}$ are unique in formula (6.14) $-\mu_{f, g}$ is not necessarily vanishing at $\{0\}$ and we get a compact formula, in which the Gaussian part can be incorporated into the Poisson one. This together with the proof of Theorem 6.1 explains in some way why Theorem 6.3 is true.

Remark 6.0. Example 6.0 shows that in general, except for instance if $X=\boldsymbol{R}^{1}$ the "weak" form (class $\mathrm{CP}(X ; \mathscr{E})$ ) of conditional positive definiteness need not imply the strong form, namely the conditional positive definiteness, although the group parameter $x$ varies over the commutative group $X$. This fact is in a strong contrast to the property that "weak" positive definiteness on commutative group $X$ implies the positive definiteness. More precisely, if for every $f \in \mathscr{E}$ the scalar function $X \times X:(x, y)$ 
$\rightarrow l_{x-y}(f, f)\left(l_{x}(f, g)\right.$ bilinear in $\left.f, g\right)$ is positive definite, then the function $l=\left\{l_{x-y}(f, g)\right\}$ is positive definite. For discussion of this matter in terms of operators, which makes no essential restriction see [22].

There is a simple way of producing of Gaussian "type" conditionally positive definite functions, originating from construction of tensor products of Hilbert spaces. Suppose namely that $(\cdot, \cdot)$ is a semi-inner product on the complex linear space $\mathscr{E}$ and $[\cdot, \cdot]$ a semi-inner product on the real space $X$. This is the trivial consequence of Schur's lemma then in this case the function

$$
(x, y) \rightarrow-G(f, g ; x-y, x-y)=-(f, g)[x-y, x-y]
$$

is conditionally positive definite. If $X$ is a real separable Hilbert space and $[x, y]=\langle B x, y\rangle$ where $B$ is an $S$-operator, then our function is $S$-continuous and consequently is a conditionally positive definite function of class $\mathrm{CP}(X ; \mathscr{E})$.

7. Structural functions of $\operatorname{CP}(X ; \mathscr{E})$ functions. This section deals with some analy tic formulae for structural functions; $X$ as before is a separable real Hilbert space and $\mathscr{E}$ a linear complex space. Later on we study the spectral picture of related unitary representations of $X$ along the general theory of Section 5. The basic result is the Yaglom formula [35] - see formula (7.7) below. Our rather general arguments and statements are direct and we do not appeal to [35], where the generalized Ito-Gelfand processes are considered, which gives the rise to "shifting" of references.

We work with the Fourier transform

$$
(F \varphi)(x)=\hat{\varphi}(x)=\frac{1}{(2 \pi)^{n / 2}} \int_{\boldsymbol{R}^{n}} e^{i\langle x, y\rangle} \varphi(y) d y
$$

for $\varphi$ in the Schwartz space $S\left(\boldsymbol{R}^{n}\right)$ of rapidly vanishing at infinity $C^{\infty}$ functions on $\boldsymbol{R}^{n}$. We refer in the matter to Simon-Reed [29] and Rudin [30]. The basic property we need is that $F S\left(\boldsymbol{R}^{n}\right)=S\left(\boldsymbol{R}^{n}\right)$. Our uniqueness and existence statements concern among others the involved essentially complex measures, which for spectral theory for $X=\boldsymbol{R}^{1}$ are needless. Complex measures is understood here as in Rudin [31] and we follow this reference in all what follows.

To begin with we will prove the following:

Lemia 7.0. Let $z_{p}(x, y)(p=1,2) x, y \in X$ be complex valued, real bilinear forms and $\mu_{p}(p=1,2)$ complex measures, vanishing on $\{0\}$. Then, if

$$
z_{1}\left(x-y, x^{\prime}-y^{\prime}\right)+\int_{X}\left(e^{i\langle x, s\rangle}-e^{i\langle y, s\rangle}\right)\left(e^{i\left\langle x^{\prime}, s\right\rangle}-e^{i\left\langle y^{\prime}, s\right\rangle}\right) \frac{1+|s|^{2}}{|s|^{2}} d \mu_{1}(s)
$$




$$
=z_{2}\left(x-y, x^{\prime}-y^{\prime}\right)+\int_{X}\left(e^{i\langle x, s\rangle}-e^{i\langle y, s\rangle}\right)\left(e^{i\left\langle x^{\prime}, s\right\rangle}-e^{i\left\langle y^{\prime}, s\right\rangle}\right) \frac{1+|s|^{2}}{|s|^{2}} d \mu_{2}(s)
$$

for all $x, y, x^{\prime}, y^{\prime} \in X$, then $z_{1}(x, y)=z_{2}(x, y)$ for $x, y \in X$.

Proof. Notice first that since $\left|e^{i u}-1\right| \leqslant|u|$ for every real $u$, then if $0<|s|<1$ then for real $t$ gives us that, since $|\langle u, v\rangle| \leqslant|u||v|$,

$$
\left|e^{i\langle t x, s\rangle}-1\right|\left|e^{-i\left\langle t x^{\prime}, s\right\rangle}-1\right| \frac{1+|s|^{2}}{|s|^{2}} \leqslant t^{2}|x|\left|x^{\prime}\right||s|^{2} \frac{1+|s|^{2}}{|s|^{2}} \leqslant 2 t^{2}|x|\left|x^{\prime}\right| .
$$

If $|s|>1$, then

$$
\left|e^{i\langle t x, s\rangle}-1\right| \sqrt{\frac{1+|s|^{2}}{|s|^{2}}} \leqslant 2 \sqrt{2}, \quad\left|e^{-\left\langle t x^{\prime}, s\right\rangle}-1\right| \sqrt{\frac{1+|s|^{2}}{|s|^{2}}} \leqslant 2 \sqrt{2} .
$$

Consequently, for $s \neq 0$

$$
r\left(t x, t x^{\prime}, s\right)=\left|\left(e^{i\langle t x, s\rangle}-1\right)\left(e^{-\left\langle t x^{\prime}, s\right\rangle}-1\right)\right| \frac{1+|s|^{2}}{|s|^{2}} \leqslant 2 t^{2}|x|\left|x^{\prime}\right|+8
$$

which, since $\mu_{1}(\{0\})=0=\mu_{2}(\{0\})$, proves that integrals appearing above make sense. The last inequality yields for $t \geqslant t_{0}>0$

$$
\frac{r\left(t x, t x^{\prime}, s\right)}{t^{2}} \leqslant 2|x|\left|x^{\prime}\right|+\frac{8}{t_{0}^{2}}
$$

for $s \neq 0$. On the other hand, for fixed $s \neq 0$ and fixed $x, x^{\prime}$ and $t \geqslant t_{0}>0$

$$
\frac{1}{t^{2}} r\left(t x, t x^{\prime}, s\right) \leqslant \frac{4}{t^{2}} \frac{1+|s|^{2}}{|s|^{2}} \quad \text { for } s \neq 0 \text {. }
$$

Since $\mu=\mu_{1}-\mu_{2}$ vanishes on the singleton $\{0\}$ and

$$
z_{1}\left(x, x^{\prime}\right)-z_{2}\left(x, x^{\prime}\right)=\int_{X} \frac{\left(e^{i\langle l x, s\rangle}-1\right)\left(e^{i\left\langle\left\langle x^{\prime}, s\right\rangle\right.}\right)}{t^{2}} \frac{1+|s|^{2}}{|s|^{2}} d \mu(s),
$$

(7.0) and (7.1) imply by dominated convergence that the right-hand of the last equality tends to zero if $t \rightarrow+\infty$, which proves the claim.

The following lemma is now in order:

LEMMA 7.1. Suppose that $X=\boldsymbol{R}^{n}$ and let $\mu$ be a complex measure on $B\left(\boldsymbol{R}^{n}\right)$, i.e. $d \mu=h d|\mu|$ where $h$ is a Borel measurable function and $|h(x)|$ $=1$ for all $x \in X$ and $|\mu|$, the total variation of $\mu$ is a finite positive measure. We assume that $\mu(\{0\})=0$, i.e. $|\mu|(\{0\})=0$. Then, if

$$
\int_{\mathbf{R}^{n}}\left(e^{i\langle u, \theta\rangle}-1\right)\left(e^{i\langle v, s\rangle}-1\right) \frac{1+|s|^{2}}{|s|^{2}} d \mu(s)=0
$$

for $u, v \in \boldsymbol{R}^{n}$, then $\mu=0$. 
Proof. Using the inequality $\left|e^{i r}-1\right| \leqslant|r|$ for real $r$ we get that, since $|\langle u, s\rangle| \leqslant|u||s|$,

$$
\left|e^{i\langle u, s\rangle}-1\right| \sqrt{\frac{1+|s|^{2}}{|s|^{2}}} \leqslant 2|u| \quad \text { if } 0<|s| \leqslant 1
$$

and

$$
\left|e^{i\langle u, s\rangle}-1\right| \sqrt{\frac{1+|s|^{2}}{|s|^{2}}} \leqslant 2 \sqrt{2} \quad \text { if }|s|>1
$$

It follows that

$$
\left|\left(e^{i\langle u, s\rangle}-1\right) \sqrt{\frac{1+|s|^{2}}{|s|^{2}}}\right| \leqslant 2(|u|+\sqrt{2}) \quad \text { for } s \neq 0,
$$

and consequently for $\varphi \in S\left(\boldsymbol{R}^{n}\right)$, for fixed $v \in \boldsymbol{R}^{n}$

$$
\int_{\boldsymbol{R}^{n}} \int_{\boldsymbol{R}^{n}}\left|\varphi(u)\left(e^{i\langle u, s\rangle}-1\right)\left(e^{i\langle 0, s\rangle}-1\right)\right| \frac{1+|s|^{2}}{|s|^{2}} d\left|\mu_{s}\right| d u<+\infty
$$

simply because $\int_{R^{n}}|\varphi(u)(|u|+\sqrt{2})| d u<+\infty$. It now follows from (7.2) and Fubini's theorem that for $v \in \boldsymbol{R}^{n}$

$$
\begin{aligned}
0 & =\int_{\boldsymbol{R}^{n}}\left[\int_{\boldsymbol{R}^{n}} \varphi(u)\left(e^{i\langle u, s\rangle}-1\right)\left(e^{i\langle v, \mathrm{~s}\rangle}-1\right) \frac{1+|s|^{2}}{|s|^{2}} d u\right] d \mu_{\mathrm{g}} \\
& =\int_{\mathbf{R}^{n}}\left[\int_{\boldsymbol{R}^{n}} \varphi(u)\left(e^{i\langle u, s\rangle}-1\right) d u \cdot\left(e^{i\langle v, s\rangle}-1\right) \frac{1+|s|^{2}}{|s|^{2}}\right] d \mu_{\mathrm{s}} \\
& =\int_{\mathbf{R}^{n}}(\hat{\varphi}(s)-\hat{\varphi}(0))\left(e^{i\langle v, s\rangle}-1\right) \frac{1+|s|^{2}}{|s|^{2}} d \mu_{\mathrm{s}} .
\end{aligned}
$$

We take a $\varphi$ such that $\hat{\varphi}(x) \stackrel{\text { df }}{=} \psi(x)$ where $\psi$ is $C^{\infty}$ and with compact support. Such $\psi$ exists, because $F S\left(\boldsymbol{R}^{n}\right)=S\left(\boldsymbol{R}^{n}\right)$. We can choose such $\psi$ which vanishes around zero and conclude then by the last equality that for $v \in \boldsymbol{R}^{\boldsymbol{n}}$

$$
\int_{\boldsymbol{R}^{n}} \psi(s) e^{i\langle v, s\rangle} \frac{1+|s|^{2}}{|s|^{2}} h d\left|\mu_{s}\right|=\int_{\boldsymbol{R}^{n}} \psi(s) \frac{1+|s|^{2}}{|s|^{2}} h d\left|\mu_{s}\right| \cdot
$$

The left-hand side is the value of the Fourier transform of the measure $d \nu=\psi(s) \frac{1+|s|^{2}}{|s|^{2}} h(s) d\left|\mu_{s}\right|$ at the point $v$ and the right-hand side si equal to the value of $F v$ at $v=0$. It follows that $v$ is concetrated on the 
singleton $\{0\}-$ but evidently $v(\{0\})=0$. This means that $v \equiv 0$. Let $\sigma$ be an open ball, $0 \in \vec{\sigma}$ and $\psi$ such that it is equal to 1 on $\sigma$ and vanishes. in a neighbourhood of zero; such a function can be obtained - see Rudin [30], 1.46 .

Since $|h(s)|=1, \frac{1+|s|^{2}}{|s|^{2}} h(s) \neq 0$ for $s \in \sigma$, which implies, since $\nu(\sigma)=0$, that $|\mu|(\sigma)=0$. The ball $\sigma$ was arbitrary, $\bar{\sigma} \neq 0$ and $|\mu|$ is a regular measure. Since $|\mu|(\{0\})=0$, we conclude that $\mu \equiv 0$.

In order to get an infinite-dimensional analogon of Lemma 7.1 we recall some known properties of cylindrical measures - in all what follows we apply notation of [16].

If $X$ is a real separable Hilbert space and $Z$ a finite-dimensional subspace of $X$ then $P_{Z}$ stands for the orthogonal projection operator of $X$ onto $Z$. The cylinder set with base $\sigma \in B(Z)$ is the subset of $X$ of the form $\left\{x \in X ; P_{Z} x \in \sigma\right\}$. Let $B^{Z}$ be the set of all cylinder sets with bases in $B(Z)$ and define $B^{0}=\bigcup_{Z} B^{Z}$, where $Z$ runs over the totality of all finite-dimensional subspaces of $X$. Then - see [16], Proposition 7.6.1:

(7.4) The smallest $\sigma$-field, i.e. that one generated by $B^{0}$, is equal to $B(X)$. It follows from (7.4) that

(7.5) If $\mu_{1}, \mu_{2}$ are two complex measures on $B(X)$ and $\mu_{1}(a)=\mu_{2}(\alpha)$ for $\alpha \in B^{0}$, then $\mu_{1}=\mu_{2}$.

For finite-dimensional subspace $Z \subset X$ and measure $\mu$ on $B(X)$ we define the projection $\mu_{Z}$ of $\mu$ on $Z$ by the formula $\mu_{Z}(\sigma)=\mu\left(P_{Z}^{-1}(\sigma)\right)$ for $\sigma \in B(Z)$.

LeMna 7.2. Let $X$ be a real separable Hilbert space and $\mu$ a complex measure on $B(X)$. If $\mu(\{0\})=0$ and

$$
\int_{X}\left(e^{i\langle u, s\rangle}-1\right)\left(e^{i\langle v, s\rangle}-1\right) \frac{1+|s|^{2}}{|s|^{2}} d \mu_{s}=0
$$

for $u, v \in X$ then $\mu \equiv 0$.

Proof. For $u, v \in Z=$ a finite-dimensional subspace of $X$, we get from (7.6) by transformation law (see Halmos [9], § 39, Th. 3) that

$$
\int_{Z}\left(e^{i\langle u, s\rangle}-1\right)\left(e^{i\langle v, s\rangle}-1\right) \frac{1+|s|^{2}}{|s|^{2}} d \mu_{Z}(s)=0
$$

which by Lemma 7.1 implies that $\mu_{Z} \equiv 0$. It follows that $\mu(\sigma)=0$ for $\sigma \in B^{0}$ which by (7.5) proves claim.

Our basic theorem now is the following one: 
Theorem 7.0. Let $X$ be a real separable Hilbert space, $\mathscr{E}$ a complex linear space and $b_{x}(f, g)$ a function of class $\mathrm{CP}(X ; \mathscr{E})$ with Levy's-Khintchine representation (6.12)

$$
b_{x}(f, g)=i u_{f, g}(x)-\frac{1}{2} G(f, g ; x, x)+\int_{x} h(x, y) d \mu_{f, \sigma} .
$$

Then the structural function $d$ of $b$ has the unique representation

$$
\begin{aligned}
a_{(x, y) ;\left(x^{\prime}, y^{\prime}\right)}(f, g)= & G\left(f, g ; x-y, x^{\prime}-y^{\prime}\right)+ \\
& +\int_{\boldsymbol{X}}\left(e^{i\langle x, s\rangle}-e^{i\langle y, s\rangle}\right) \overline{\left(e^{i\left\langle x^{\prime}, s\right\rangle}-e^{i\left\langle y^{\prime}, s\right\rangle}\right)} \frac{1+|s|^{2}}{|s|^{2}} d \mu_{f, g}
\end{aligned}
$$

for $x, y, x^{\prime}, y^{\prime} \in X$ and $f, g \in \mathscr{E}$.

Proof. That $d$ has the representation as in (7.7) follows from Theorem 6.0. The uniqueness follows immediately from Lemma 7.0 and Lemma 7.2 by taking $y=y^{\prime}=0$.

Formula (7.7) will be called the Yaglom formula for structural function $d$ of $b$. A few comments are now in order. First, by taking $X=\boldsymbol{R}^{1}$, having in view Theorem 6.3 and the result of Masani [19], who proved in fact that every helix in $\boldsymbol{R}^{n}$ is a structural function of a conditionally positive definite scalar valued function of class $\operatorname{CP}\left(\boldsymbol{R}^{n} ; \boldsymbol{C}^{1}\right)$, we get $(\gamma)$ of Theorem 3.2 of [18] for $n=1$. Next (7.7) applies to any $b$ of $\operatorname{class} \operatorname{CP}(X ; \mathscr{E})$ which one, as shows Example 6.0, is essentially broader than the class of conditionally positive definite functions of class $\operatorname{CP}(X ; \mathscr{E})$. Finally, formula (7.7) holds for infinite-dimensional $X$ and arbitrary $\mathscr{E}$; the classical case in [18], [35] is just when $\mathscr{E}=\boldsymbol{C}^{\mathbf{1}}, X=\boldsymbol{R}^{n}$.

Suppose now that our $b=\left\{b_{x}(f, g)\right\}$ of class $\mathrm{CP}(X ; \mathscr{E})$ is conditionally positive definite, which simply means by Theorem 6.2 that the Gaussian part $-\frac{1}{2} G$ is conditionally positive definite. We are then able to apply the general theory of Section 5 which corresponds to "time domain analysis". It follows then that for $e^{x_{0}}, K_{x_{0}}, Y_{x_{0}}$ which are associated with $x_{0}=0$ there is an $S$-continuous unitary representation $\pi_{0}(s)=U_{s}$ of $X$ into $L\left(K_{0}\right)$. By Remark 5.4 we get:

(7.8) If $b=\left\{b_{x, y}(f, g)\right\}$ of class $\operatorname{CP}(X ; \mathscr{E})$ is conditionally positive definite (this happens when $X=\boldsymbol{R}^{1}$ ) then $U_{s}$ is a trivial representation, if $b$ reduces to its Gaussian part.

Having in view Theorem 6.1, it becomes natural to examine now the structural function of a function of class $\operatorname{CP}(X ; \mathscr{E})$ reducing to its Poisson part. This will be the subject in investigations we have to go through now in a spirit of "spectral domain analysis". Notice by the way, that the elementary part of functions of class $\operatorname{CP}(X ; \mathscr{E})$ have identically zero structural function, so there is no need to think about them at all. The 
role of this parts is explained via the notion of cohomologous first order cocycles - see [8], [27]; and for our analytical investigation there is no interest at this stage to take care on this parts.

To begin with we notice that for $s \neq 0$

$$
\left|e^{i\langle u, s\rangle}-1\right| \sqrt{\frac{1+|s|^{2}}{|s|^{2}}} \leqslant \sqrt{2}(|u|+2) .
$$

Let $\eta(s)$ be an $B(X)$ measurable complex function such that $\eta(0)=0$ $=$ an arbitrary number and $|\eta(s)|^{2}=\left(1+|s|^{2}\right) /|s|^{2}$ for $s \neq 0$.

LEMMA 7.3. Let $\mu$ be a complex measure on $B(X)$ such that $\mu(\{0\})=0$ If

$$
\int_{x}\left(e^{i\langle u, s\rangle}-1\right) \eta(s) d \mu_{s}=0
$$

for all $u \in X$, then $\mu \equiv 0$.

Proof. The above integral makes sense, because by (7.9) $\mid\left(e^{i\langle u, s\rangle}-\right.$ $-1) \eta(s) \mid \geqslant \sqrt{2}(|u|+2), s \neq 0$ for $u \in X$ and $\mu(\{0\})=0$. Suppose $X=\boldsymbol{R}^{n}$ and take $\varphi \in S\left(\boldsymbol{R}^{n}\right)$ such that $\hat{\varphi}(s)=\psi(s)$ is vanishing around zero. By Fubini's theorem and (7.9) we get that

$$
\begin{aligned}
\int_{\boldsymbol{R}^{n}} \int_{\boldsymbol{R}^{n}}\left(\varphi(u) e^{i\langle u, s\rangle}-1\right) \eta(s) d \mu_{s} d u & =\int_{\boldsymbol{R}^{n}}\left[\int_{\boldsymbol{R}^{n}} \varphi(u)\left(e^{i\langle u, s\rangle}-1\right) d u\right] \eta(s) d \mu_{s} \\
& =\int_{\boldsymbol{R}^{n}}(\psi(s)-\psi(0)) \eta(s) d \mu_{s}=0 .
\end{aligned}
$$

Since $\psi(0)=0$, we get therefore

$$
\int_{\mathbf{R}^{n}} \psi(s) \eta(s) d \mu_{s}=0
$$

Let $\sigma_{1} \subset \sigma_{2}$ be two closed balls in $\boldsymbol{R}^{n}$, with the common center $a$ and such that $\sigma_{1} \subset$ int $\sigma_{2}$ and $0 \notin \sigma_{2}$. We take, following [30], $1.46 \psi(s)=1-$ $-g\left(|s-a|^{2}\right)$, where $g \in C^{\infty}\left(\boldsymbol{R}^{1}\right)$ and $g(u)=0$ if $u<\sqrt{r_{1}}, g(u)=1$ if $u>\sqrt{r_{2}}$, where $r_{i}=$ radius of $\sigma_{i}(i=1,2)$. We can take $g$ so that without any loss of generality $|g(u)| \leqslant 1$ for all $u$. It follows then that $|\psi(s)| \leqslant 2$ for all $s$ and any $\sigma_{1}, \sigma_{2} ; \psi(s)=1$ on $\sigma_{1}$ and $\psi(s)=0$ outside of $\sigma_{2}$. Formula (7.10) yields that

$$
\int_{\sigma_{1}} \eta(s) d \mu_{s}+\int_{\sigma_{2}-\sigma_{1}} \psi(s) \eta(s) d \mu_{s}=0 .
$$

Let us take a decreasing sequence $\sigma_{2}^{(v)}$ such that $\sigma_{2}^{(v)} \rightarrow \sigma_{1}$. Then

$$
\left|\int_{\sigma_{2}^{(v)}-\sigma_{1}} \psi(s) \eta(s) d \mu_{s}\right| \leqslant 2 \sup _{\substack{\sigma_{2}^{(v)}-\sigma_{1} \\ \mid}}|\eta(s)||\mu|\left(\sigma_{2}^{(v)}-\sigma_{1}\right) \rightarrow 0
$$


which by (7.11) with $\sigma_{2}=\sigma_{2}^{(v)}$ proves that $\int_{\sigma_{1}} \eta(s) d \mu_{s}=0$. Since $\sigma_{1}$ was an arbitrary closed ball not including zero, $\mu(\{0\})=0$ and $\mu$ is regular, we conclude, since $\eta(s) \neq 0$ for $s \neq 0$, that $\mu \equiv 0$.

Just like in the proof of Lemma 7.2 we conclude now that $\mu \equiv 0$ for any real separable Hilbert space $X$.

Now suppose that the function $b_{x}(f, g)$ is of Poisson type, i.e.

$$
b_{x}(f, g)=\int_{X}\left(e^{i\langle x, v\rangle}-1-\frac{i\langle x, y\rangle}{1+|y|^{2}}\right) \frac{1+|y|^{2}}{|y|^{2}} d \mu_{f, \theta}(y),
$$

where $\mu_{f, \sigma}$ are such as in Theorem 6.0. Since $\mu_{f, f}(\sigma) \geqslant 0$ for $\sigma \in B(X)$, we can apply Proposition 2.1 and just get that $\mu_{f, \theta}(\sigma)=(E(\sigma) R f, R g)_{K}$, where $K$ is a complex Hilbert space, $E(\cdot)$ a spectral measure on $K$ and $R: \mathscr{E} \rightarrow K$ a linear operator. We assume once for all that $\boldsymbol{K}$ is minimal, i.e. $K=\bigvee_{\sigma \in B(X)} E(\sigma) R \mathscr{E}$ what determines in a unique way $K, E, R$ up to unitary isomorphism and the formula

$$
\mu_{f, g}\left(\sigma_{1} \cap \sigma_{2}\right)=\left(E\left(\sigma_{1}\right) R f, E\left(\sigma_{2}\right) R g\right)
$$

is the corresponding canonical representation of function $\left\{\sigma_{1}, \sigma_{2}\right\} \rightarrow \mu_{f, 0}\left(\sigma_{1} \cap\right.$ $\left.\cap \sigma_{2}\right)$. Notice that $E(\{0\})=0$, because all measures $\mu_{f, \theta}$ vanish on $\{0\}$. It follows now from (7.9) that for every $u$ and $\eta$ such as in Lemma 7.3, the function $h_{u}(s)=\left(e^{i\langle u, s\rangle}-1\right) \eta(s)$ for $s \neq 0, h_{u}(0)=c$ (any number) is $B(X)$ measurable and $E$-bounded, simply because it is bounded. It follows then that if $\eta(s)$ is a $B(X)$ complex valued function such that $|\eta(s)|^{2}=\left(1+|s|^{2}\right) /|s|^{2}$ for $s \neq 0, \eta(0)=c$, then the spectral integral $\int_{X}\left(e^{i\langle x, s\rangle}-1\right) \eta(s) d E_{s}$ is a bounded linear operator in $K$.

We now define for $x \in X, f \in \mathscr{E}$

$$
Y_{\eta}(x) f=\int_{X}\left(e^{i\langle x, s\rangle}-1\right) \eta(s) d E_{s} R f
$$

$Y_{\eta}(x)$ is a linear operator from $\mathscr{E}$ into $K$.

Suppose now that $K \ni h \perp Y_{\eta}(x) f$ for $x \in X, f \in \mathscr{E}$. It follows that

$$
\int_{\bar{X}}\left(e^{i\langle x, s\rangle}-1\right) \eta(s) d\left(E_{s} R f, h\right)_{K}=0
$$

for every $x \in X$. By Lemma 7.3 we conclude that $(E(\sigma) R f, h)_{K}=0$ for every $\sigma \in B(X)$, which, since $K$ is minimal, proves that $h=0$. Consequently, since plainly $Y_{\eta}(0) f=0$ for every $f \in \mathscr{E}$, we get that

$$
K=\bigvee_{x, y \in \mathcal{X}}\left(Y_{\eta}(x)-Y_{\eta}(y)\right) \mathscr{E} \text {. }
$$

Let us now take the inner product $\left(Y_{\eta}(x) f, Y_{\eta}(y) g\right)_{K}$ for $x, y \in X, f, g \in \mathscr{E}$. 
We have then (omitting the subscript " $K$ ")

$$
\begin{aligned}
\left(Y_{\eta}(x) f, Y_{\eta}(y) g\right) & \\
= & \left(\int_{\dot{X}}\left(e^{i\langle x, s\rangle}-1\right) \eta(s) d E_{s} R f, \int_{X}\left(e^{i\langle y, u\rangle}-1\right) \eta(u) d E_{u} R g\right) \\
& =\int_{X}\left(e^{i\langle x, s\rangle}-1\right) \overline{\left(e^{i\langle y, s\rangle}-1\right)} \frac{1+|s|^{2}}{|s|^{2}} d\left(E_{s} R f, R g\right),
\end{aligned}
$$

because $E$ is a spectral measure. By the Yaglom formula we now conclude that, $d$ being the structural function of $b$,

$$
\begin{aligned}
\left(Y_{\eta}(x) f, Y_{\eta}(y) g\right) & =d_{(x, 0) ;(y, 0)}(f, g) \\
& =b_{x-y}(f, g)-b_{x}(f, g)-b_{-y}(f, g)=c_{x, y}^{x_{0}}(f, g)
\end{aligned}
$$

with $x_{0}=0$. Having in view (7.13), we infer therefore that $c_{x, y}^{x_{0}}, K, Y_{\eta}$ are associated to $x_{0}=0$ for function $b_{x}(f, g)$.

It follows then that the corresponding map

$$
\pi_{0}(x)\left(Y_{\eta}(x)-Y_{\eta}(y)\right) f=\left(Y_{\eta}(x+s)-Y_{\eta}(y+s)\right) f
$$

depending apriori on $\eta$ is an $S$-continuous unitary representation of $X$. Let us write for brevity $U_{s}$ in place of $\pi_{0}(s)$. We will show that $U_{s}=$ $\int_{X} e^{i\langle s, u\rangle} d E_{u}$, i.e. that $U_{s}$ does not depend on the choice of $\eta$. So, suppose that, by Stone's theorem,

$$
U_{s}=\int_{X} e^{i\langle s, u\rangle} d F_{u} \quad \text { for } s \in X,
$$

where $F$ is a spectral measure in $K$. It follows from the definition of $U_{s}$ $=\pi_{0}(s)$ that for $x \in X, f \in \mathscr{E}$

$$
U_{s} \bar{Y}_{\eta}(x) f=\int_{X} e^{i\langle s, y\rangle}\left(e^{i\langle x, y\rangle}-1\right) \eta(y) d E_{y} R f
$$

On the other hand,

$$
U_{s} Y_{\eta}(x) f=\int_{X} e^{i\langle s, y\rangle} d F_{y} Y_{\eta}(x) f
$$

and consequently, for every $h \in K$ and $s \in X$

$$
\int_{X} e^{i\langle s, y\rangle}\left(e^{i\langle x, y\rangle}-1\right) \eta(y) d\left(E_{y} R f, h\right)=\int_{X} e^{i\langle s, y\rangle} d\left(\boldsymbol{F}_{y} Y_{\eta}(x) f, h\right) .
$$

The uniqueness property of Fourier transforms implies now that the measures

$$
\left(e^{i\langle x, y\rangle}-1\right) \eta(y) d\left(E_{y} R f, h\right), \quad d\left(F_{y} Y_{\eta}(x) f, h\right)
$$


are equal for all $x \in X$. It follows then that for $\sigma \in B(X)$ and $x \in X$

$$
\begin{aligned}
\left(F(\sigma) Y_{\eta}(x) f, h\right) & =\left(Y_{\eta}(x) f, F^{\prime}(\sigma) h\right) \\
& =\int_{X}\left(e^{i\langle x, s\rangle}-1\right) \eta(s) d\left(E_{s} R f, F(\sigma) h\right) \\
& =\int_{\sigma}\left(e^{i\langle x, s\rangle}-1\right) \eta(s) d\left(E_{s} R f, h\right) \\
& =\int_{X}\left(e^{i\langle x, s\rangle}-1\right) \eta(s) d\left(E_{y} R f, E(\sigma) h\right) .
\end{aligned}
$$

The measures $\left(E(\alpha) R f, F^{\prime}(\sigma) h\right)$ and $(E(\alpha) R f, E(\sigma) h)$ as a function of $\alpha \in B(X)$ vanish at $\alpha=\{0\}$. Applying Lemma 7.3 to the difference of these two measures, we conclude that

$$
(E(\alpha) R f, F(\sigma) h)=(E(\alpha) R f, E(\sigma) h) \quad \text { for } \quad \alpha \in B(X)
$$

which, since $K$ is minimal, implies that $F(\sigma)=E(\sigma)$ for $\sigma \in B(X)$.

We just obtained the spectral picture of spaces and representations related to Poisson type functions of class $\mathrm{CP}(X ; \mathscr{E})$, which by Theorem 6.1 is conditionally positive definite.

Summing up we get the following theorem:"

THeOREM 7.1. Let $\left\{b_{x}(f, g)\right\}$ be a function of $\mathrm{CP}(X ; \mathscr{E})$ class of Poisson type and

$$
b_{x}(f, g)=\int_{\dot{X}}\left(e^{i\langle x, v\rangle}-1-\frac{i\langle x, y\rangle}{1+|y|^{2}}\right) \frac{1+|y|^{2}}{|y|^{2}} d \mu_{f, g}
$$

its Levy-Khintchine canonical representation. Suppose $\mu_{f, \theta}(\sigma)=(E(\sigma) R f$, $R g)_{K}$ is the canonical representation of the related family of measures $\mu_{f, g}$.

For every complex $B(X)$ measurable function $\eta(s)$ such that $|\eta(s)|^{2}$ $=\left(1+|s|^{2}\right) /|s|^{2}$ for $s \neq 0, \eta(0)=c$ (arbitrary complex number) the formula $Y_{\eta}(x) f=\int_{X}\left(e^{i\langle x, s\rangle}-1\right) \eta(s) d E_{s} R f$ defines a family of linear maps from $\mathscr{E}$ into $K$, such that $c_{x, y}^{0}(f, g)=b_{x-y}(f, g)-b_{x}(f, g)-b_{-y}(f, g), K$ and $Y$ are associated to $x_{0}=0$, i.e.

$$
\begin{gathered}
K=\bigvee_{\sigma \in B(X)} E(\sigma) R \mathscr{E}=\bigvee_{x, y \in X}\left(Y_{\eta}(x)-Y_{\eta}(y)\right) \mathscr{E}, \\
c_{x, y}^{0}(f, g)=\left(Y_{\eta}(x) f, Y_{\eta}(y) g\right)_{K} \quad \text { for } x, y \in X ; f, g \in \mathscr{E} .
\end{gathered}
$$

Moreover, the mapping $U_{s} Y_{\eta}(x) f=\left(Y_{\eta}(x+s)-Y_{\eta}(s)\right) f$ defines an S-continuous unitary representation of the additive group of $X, U_{s}$ does not depend on $\eta$ and has the spectral representation

$$
U_{s}=\int_{X} e^{i\langle x, y\rangle} d E_{y}
$$

15 - Annales Polonici Mathematicl XuII 
Remark 7.0. Notice that canonical representations related to $Y_{\eta}$ corresponds to the same space $\boldsymbol{K}$. So, we have an natural example of different canonical representations with the fixed minimal space. It follows that for $\eta_{1}, \eta_{2}$ there is a unitary map $U_{\eta_{1}, \eta_{2}}$ such that $U_{\eta_{1}, \eta_{2}} \bar{\eta}_{\eta_{2}}(x) f$ $=Y_{\eta_{1}}(x) f$ for $x \in X, f \in \mathscr{E}$. It is easy to show that $U_{\eta_{1}, \eta_{2}}$ commute with $U_{s}$, i.e. with $E(\cdot)$. We observe by the way that there is a lot of functions $\eta$ satisfying the relation

$$
\begin{gathered}
|\eta(s)|^{2}=\frac{1+|s|^{2}}{|s|^{2}} \quad \text { for } s \neq 0 \text { - for example } \eta(s)=\frac{i \pm|s|}{|s|}, \\
\eta(s)= \pm \sqrt{\frac{1+|s|^{2}}{|s|^{2}}} \\
\eta(s)=e^{i a} \varrho(s) \quad(a \text { real }), \quad \text { where } \quad|\varrho(s)|^{2}=\frac{1+|s|^{2}}{|s|^{2}}, \text { etc. }
\end{gathered}
$$

We can take measurable partitions of $X$ and define on its parts $\eta(s)$ by different formulas, for instance by the ones given above.

COROLLARY 7.0. If $X=\boldsymbol{R}^{\mathbf{l}}$, then, as we notice already, the Gaussian part of $\operatorname{CP}\left(\boldsymbol{R}^{1} ; \&\right)$ can be "swallowed" by the Poisson part - see (6.14) and comments after Theorem 7.0. It is then possible to get an analogon of our Theorem 7.1 when adding the Gaussian part, whose classical model scalar version for $\eta(s)=(i+s) / s$ is due to Masani [18]. The suitable uniqueness statement follows from uniqueness properties which we proved in this section. The corresponding operator version of Masani's theorem will be given in the next section.

CoROLLARY 7.1. The closed support $S_{E}$ of the spectral measure $E(\cdot)$ is called the Poisson, spectrum for any $b$, whose Poisson part is as in canonical representation of $b=i u-\frac{1}{2} G+P$. Suppose $b=P$. If 0 is not in $S_{E}$, then $E(\sigma)=0$ for some neighbourhood $\sigma$ of 0 . Using notation of the above theorem, the formula

$$
F(a)=\int_{a} \frac{1+|y|^{2}}{|y|^{2}} d E_{y}
$$

defines a semi-spectral measure on $K$, for $\alpha \in B(X)$.

Using Proposition 2.0 for $v_{f, g}(a)=(F(a) f, g)_{K}$ or, which is equivalent in this case, the classical Naimark's theorem - see [22]. [24], we conclude that there is a complex Hilbert space $\tilde{K}$, a spectral measure $\tilde{E}(\cdot)$ in $\tilde{K}$ and a continuous linear operator $\nabla: K \rightarrow \tilde{K}$ such that $F(\alpha)=V^{*} \tilde{E}(\alpha) V$ for $\alpha \in B(X)$ - the minimality condition $\tilde{\boldsymbol{K}}=\bigvee \tilde{\boldsymbol{E}}(\alpha) V \boldsymbol{K}$ determines $\boldsymbol{K}, \boldsymbol{\nabla}, \boldsymbol{E}$ up to unitary isomorphism. Having $b$ of the Poisson type as in Theorem 7.1 
qoe define

$$
u_{f, g}(x)=-\int_{X} \frac{\langle x, y\rangle}{|y|^{2}} d v_{f, g}(y)
$$

Since

$$
b_{x}(f, g)=\int_{X}\left(e^{i\langle x, y\rangle}-\frac{i\langle x, y\rangle}{1+|y|^{2}}\right) \frac{1+|y|^{2}}{|y|^{2}} d \mu_{f, g}(y)
$$

and $\left(\frac{d \mu_{f, 0}}{d v_{f, \theta}}\right)(y)=\frac{|y|^{2}}{1+|y|^{2}}$ for $v_{f, \theta}$ (or, as it is equivalent to $\mu_{f, \theta}$ ) almost all $y \in X$, we get

$$
\begin{aligned}
b_{x}(f, g) & =u_{f, \theta}(x)+\int_{\tilde{X}}\left(e^{i\langle x, y\rangle}-1\right) d v_{f, g}(y) \\
& =u_{f, \theta}(x)+\int_{\tilde{X}}\left(e^{i\langle x, y\rangle}-1\right) d\left(\tilde{E}_{y} \nabla R f, \nabla R g\right)_{\tilde{K}}
\end{aligned}
$$

which implies for any $b$ of class $\operatorname{CP}(X ; \mathscr{E})$ such that $0 \notin S_{E}$ the formula

$$
\begin{aligned}
b_{x}(f, g)=u_{f, g}(x)-\frac{1}{2} G(f, g ; & x, x)+ \\
& +\int_{\tilde{X}}\left(e^{i\langle x, y\rangle}-1\right) d\left(\tilde{\boldsymbol{E}}_{\boldsymbol{\nu}} \boldsymbol{\nabla} \boldsymbol{R} f, \boldsymbol{\nabla} \boldsymbol{R}\right)_{\boldsymbol{K}}
\end{aligned}
$$

which, following Cuppens [3], Theorem 4.3.1, we call the De Finnetti formula. The structural function $d$ of $b$ is of the form

$$
\begin{aligned}
& d_{(x, y) ;\left(x^{\prime}, y^{\prime}\right)}(f, g)=G\left(f, g ; x-y, x^{\prime}-y^{\prime}\right)+ \\
& \quad+\int_{X}\left(e^{i\langle x, s\rangle}-e^{i\langle y, s\rangle}\right) \overline{\left(e^{i\left\langle x^{\prime}, s\right\rangle}-e^{i\left\langle y^{\prime}, s\right\rangle}\right)} d v_{f, g}(s)
\end{aligned}
$$

and the spectral domain picture for $P=b$ in this case, associated to $x_{0}=0$ takes the simple form for $\tilde{\boldsymbol{B}} \stackrel{\mathrm{d}}{=} \nabla R$ :

$$
\tilde{\boldsymbol{K}}=\bigvee_{a \in B(X)} \tilde{\boldsymbol{E}}(a) \tilde{R} \mathscr{E}, \quad \tilde{Y}_{0}(x) f=\int_{X}\left(e^{i\langle x, y\rangle}-1\right) d \tilde{E} \tilde{\boldsymbol{X}}_{y} \tilde{R} f
$$

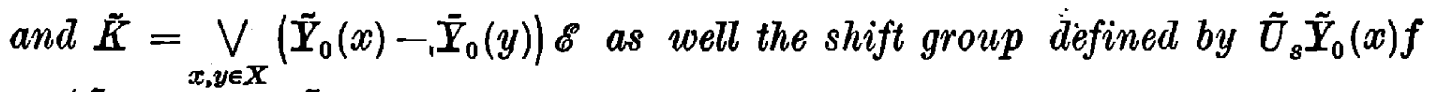
$=\left(\tilde{Y}_{0}(x+s)-\bar{Y}_{0}(s)\right) f$ has the spectral representation

$$
\tilde{U}_{s}=\int_{\tilde{X}} e^{i\langle x, y\rangle} d \tilde{E}_{y} .
$$

The proofs of all these statements are just as in the proof of Theorem 7.1, with obviously simplified versions of previous lemmas, for we have not "fight" with the factor $\left(1+|y|^{2}\right) /|y|^{2}$, when $y$ ranges close to zero. Recall that all this works provided $0 \notin S_{E}$, i.e. there is a neighbourhood 
$\sigma$ of 0 such that all measures $\mu_{f, f}(\sigma)$ appearing in the Levy-Khintchine canonical representation of $b$ vanish on $\sigma$.

8. Continuity properties. In this section we assume once for all that $X=\boldsymbol{R}^{m}$ for some $m$. If $b_{x}^{(v)} \in \operatorname{CP}\left(X ; C^{1}\right), b_{x}^{(0)} \in \operatorname{CP}\left(X ; C^{1}\right)$, i.e. if $b_{x}^{(v)}, b_{x}^{(0)}$ are scalar valued conditionally positive definite functions and for $v$ $=0,1, \ldots$

$$
b_{x}^{(v)}=i\left\langle u^{(v)}, x\right\rangle-\frac{1}{2}\left\langle B_{v} x, x\right\rangle+\int_{R^{m}}\left(e^{i\langle x, y\rangle}-1-\frac{i\langle x, y\rangle}{1+|y|^{2}}\right) \frac{1+|y|^{2}}{|y|^{2}} d \mu_{v},
$$

the convergence $b_{x}^{(v)} \rightarrow b_{x}^{(0)}$ for $x \in \boldsymbol{R}^{m}$ does not imply that $B_{v} \rightarrow B_{0}$ and that $\mu_{v}$ converge weakly or in some other reasonable way to $\mu_{0}$. The reason is that (see [3], Theorem 4.2.4) every infinitely divisible distribution over $\boldsymbol{R}^{m}$ is a complete limit of a sequence of finite convolutions of Poisson type distributions. However, if we have to do with $\operatorname{CP}\left(\boldsymbol{R}^{m}, \mathscr{E}\right)$ functions the situation is not so bad. The "philosophical" reason is that functions of this class are in some sense rigid, for they satisfy for example some consistency conditions following from the bilinearity in $f, g \in \mathscr{E}$. To make it clear let us take $\mathscr{E}=C^{P}$ with the natural convergence in $C^{P}$ and let $b_{x}(f, g)$ be of class $\operatorname{CP}\left(\boldsymbol{R}^{m} ; \boldsymbol{C}^{P}\right)$ with canonical representation

$$
\begin{aligned}
b_{x}(f, g)=u_{f, g}(x)-\frac{1}{2} G & (f, g ; x, x)+ \\
& +\int_{\boldsymbol{R}^{m}}\left(e^{i\langle x, y\rangle}-1-\frac{i\langle x, y\rangle}{1+|y|^{2}}\right) \frac{1+|y|^{2}}{|y|^{2}} d \mu_{f, g}(y) .
\end{aligned}
$$

Let $e_{1}, \ldots, e_{p}$ be the orthogonal basis in $C^{p}$ and suppose that $f_{n} \rightarrow f_{0}$, $g_{n} \rightarrow g_{0}$. If

$$
f_{n}=\sum_{j \mid 1}^{p} a_{j, n} e_{j}, \quad g_{n}=\sum_{j \mid 1}^{p} \beta_{j, n} e_{j} \quad \text { for } n=0,1, \ldots,
$$

then for $x \in X=\boldsymbol{R}^{m}$

$$
\begin{aligned}
& b_{x}\left(f_{n}, g_{n}\right)= \sum_{j \mid 1}^{p} \sum_{k \mid 1}^{p} \alpha_{j, n} \overline{\beta_{j, n}} u_{e_{j}, e_{k}}(x)-\frac{1}{2} \sum_{j, k \mid 1}^{p} \alpha_{j, n} \overline{\beta_{k, n}} G\left(e_{j}, e_{k} ; x, x\right)+ \\
&+\sum_{j, k \mid 1}^{p} \int_{R^{m}}\left(e^{i\langle x, y\rangle}-1-\frac{i\langle x, y\rangle}{1+|y|^{2}}\right) \frac{1+|y|^{2}}{|y|^{2}} a_{j, n} \overline{\beta_{k, n}} d \mu_{e_{j}, e_{k}}(y) \\
& \underset{n \rightarrow \infty}{\longrightarrow} \sum_{j, k \mid 1}^{p} a_{j, 0} \overline{\beta_{k, 0}} u_{e_{j}, e_{k}}(x)-\frac{1}{2} \sum_{j, k \mid 1}^{p} \alpha_{j, 0} \overline{\beta_{k, 0}} G\left(e_{j}, e_{k} ; x, x\right)+ \\
&+\sum_{j, k \mid 1}^{p} \int_{x}\left(e^{i\langle x, y\rangle}-1-\frac{i\langle x, y\rangle}{1+|y|^{2}}\right) \frac{1+|y|^{2}}{|y|^{2}} a_{j, 0} \overline{\beta_{k, 0}} d \mu_{e_{j}, e_{k}}(y)=b_{x}\left(f_{0}, g_{0}\right)
\end{aligned}
$$


for every $x$, and it is plain that the elementary (Gaussian, Poisson resp.) parts of $b_{x}\left(f_{n}, g_{n}\right)$ converge to elementary (Gaussian, Poisson resp.) parts of $b_{x}(f, g)$. The moral is that the situation is not hopeless in contrary to what we said before. Notice that if $\mathscr{E}=C^{1}$ the example above becomes pretty trivial, because $b_{x}\left(f_{n}, g_{n}\right)=b_{x}(1,1) f_{n} \bar{g}_{n}$ in this case, as well $G\left(f_{n}, g_{n}\right.$; $x, x)=G(1,1 ; x, x) f_{n} \bar{g}_{n}, \quad P\left(f_{n}, g_{n} ; x\right)=P(1,1 ; x) f_{n} \bar{g}_{n} \quad$ and $\quad u_{f_{n}, \sigma_{n}}(x)$ $=u_{1,1}(x) f_{n} \bar{g}_{n}$.

In order to be in the general investigation we will prove the "well-known" inequality:

LeMma 8.0. For every $m=1,2, \ldots$, for every $h>0$ there is a constant $\eta_{m}(h)>0$ such that the inequality

$$
0<\eta_{m} \leqslant\left(1-\prod_{k \mid 1}^{m} \frac{\sin h y_{k}}{h y_{k}}\right) \frac{1+|y|^{2}}{|y|^{2}}=s_{h}(y)
$$

holds true for every $y=\left(y_{1}, \ldots, y_{m}\right) \neq 0, y \in \boldsymbol{R}^{m},\left(\frac{\sin 0}{0}=1\right.$ by definition $)$.

Proof. Suppose our assertion is not true. Then there is a sequence $y_{v}=\left(y_{1}^{(v)}, \ldots, y_{m}^{(0)}\right) \neq 0$ such that for some $h>0$

$$
s_{h}\left(y_{v}\right) \rightarrow 0 \text {. }
$$

Choosing a subsequence of $\left\{y_{v}\right\}$ if necessary, we have only three possibilities:

(i) $\left|y_{v}\right| \rightarrow \infty$,

(ii) $\left|y_{v}\right| \rightarrow 0$,

(iii) $y_{v} \rightarrow y_{0} \neq 0$.

In case (i) $\left|y_{k_{0}}^{(v)}\right| \rightarrow \infty$ for some $k_{0}$.

Hence $\prod_{k \mid 1} \frac{\sin h y_{k}^{(v)}}{h y_{k}^{v}} \underset{v \rightarrow 0}{\longrightarrow} 0$, because $\left|\frac{\sin u}{u}\right|<1$ for $u \neq 0$ which implies that $s_{h}\left(y_{v}\right) \rightarrow 1$ which is in contradiction with (8.1). In case (ii) our arguments go as follows: since $1-\cos u \geqslant u^{2} / 2$ ! $-u^{4} / 4$ ! for every real $u$, we have

$$
(1-\cos \langle x, y\rangle) \geqslant \frac{\langle x, y\rangle^{2}}{2 !}-\frac{\langle x, y\rangle^{4}}{4 !}
$$

for $x, y \in \boldsymbol{R}^{n}$. Notice now that (see Section 6 ) for $h>0$

$$
\left(1-\prod_{k \mid \mathfrak{1}}^{m} \frac{\sin h y_{k}}{h y_{k}}\right)=\frac{1}{(2 h)^{m}} \int_{\boldsymbol{K}_{h}}(1-\cos \langle x, y\rangle) d x
$$

where $K_{h}=\left\{y=\left(y_{1}, \ldots, y_{m}\right):\left|y_{i}\right| \leqslant h\right.$ for $\left.i=1,2, \ldots, m\right\}$ simply, because

$$
(1-\cos \langle x, y\rangle)=\operatorname{Re}\left(1-e^{i\langle x, y\rangle}\right)
$$


and

$$
\int_{K_{\eta}} e^{i\langle y, x\rangle} d x=\prod_{k \mid 1}^{m} \int_{-h}^{+h} e^{i x_{k} y_{k}} d x_{k}=(2 h)^{m} \prod_{k \mid 1}^{m} \frac{\sin h y_{k}}{h y_{k}} .
$$

It follows now from (8.2) that

$$
s_{h}\left(y_{v}\right) \geqslant \frac{1}{(2 h)^{m}} \frac{1+\left|y_{v}\right|^{2}}{\left|y_{v}\right|^{2}}\left(J_{1}\left(y_{v}\right)-J_{2}\left(y_{v}\right)\right)
$$

where

$$
J_{1}(y)=\int_{s_{h}} \frac{\langle x, y\rangle^{2}}{2 !} d x, \quad J_{2}(y)=\int_{s_{h}} \frac{\langle x, y\rangle^{2}}{4 !} d x
$$

and $S_{h}=\{x:|x| \leqslant h\} \subset K_{h}$. Since in general $\int_{S_{1}}\langle x, y\rangle^{2} d x=\frac{\sqrt{\pi^{m}}}{2 \Gamma\left(\frac{1}{2} m+2\right)}|y|^{2}$, we have

$$
J_{1}(y)=\frac{h^{m+2} \sqrt{\pi^{m}}}{4 \Gamma\left(\frac{1}{2} m+2\right)}|y|^{2}=\varrho|y|^{2}
$$

where $\varrho$ is the above coefficient at $|y|^{2}$. It follows now that

$$
s_{h}\left(y_{v}\right) \geqslant \frac{1}{(2 h)^{m}}\left(1+\left|y_{v}\right|^{2}\right)\left(\varrho-\frac{1}{\left|y_{v}\right|^{2}} J_{2}\left(y_{v}\right)\right)
$$

But

$$
\frac{1}{\left|y_{v}\right|^{2}} J_{2}\left(y_{v}\right)=\frac{1}{4 !} \int_{s_{h}} \frac{\left\langle x, y_{v}\right\rangle^{4}}{\left|y_{v}\right|^{2}} d x \underset{v \rightarrow \infty}{\longrightarrow} 0
$$

because $\frac{|\langle x, y\rangle|^{4}}{|y|^{2}} \leqslant \frac{|y|^{4}|x|^{4}}{|y|^{2}}=|y|^{2}\left|x^{2}\right|$ for $y \neq 0$. It follows now from (8.1) and (8.3) that

$$
0=\lim _{v \rightarrow \infty} s_{h}\left(y_{v}\right) \geqslant \frac{\because \varrho}{(2 h)^{m}}>0
$$

which is a nonsens.

Suppose (iii) holds true. Then $s_{h}\left(y_{v}\right) \rightarrow s_{h}\left(y_{0}\right)$. Since $y_{0} \neq 0$ then, since $\left|\frac{\sin u}{u}\right|<1$ for $u \neq 0$

$$
\begin{aligned}
s_{h}\left(y_{0}\right) & =\left(1-\prod_{k \mid 1}^{m} \frac{\sin h y_{k}^{(0)}}{h y_{k}^{(0)}}\right) \frac{1+\left|y_{0}\right|^{2}}{\left|y_{0}\right|^{2}} \\
& >\left(1-\prod_{k \mid 1}^{m}\left|\frac{\sin h y_{k}^{(0)}}{h y_{k}^{(0)}}\right|\right) \frac{1+\left|y_{0}\right|^{2}}{\left|y_{0}\right|^{2}}>0
\end{aligned}
$$

which is in contradiction with (8.1). 
We next formulate (see [3], Chapter 2 for references) a consequence 'of Levy's continuity theorem for characteristic functions of probability distributions:

(L) Let $\nu_{n}$ be a sequence of probability measures on $B\left(\boldsymbol{R}^{m}\right)$ and $\hat{\nu}_{n}(x)$ their characteristic function. Let $\nu$ be a probability measure on $B\left(\boldsymbol{R}^{m}\right)$ such that for $x \in \boldsymbol{R}^{m} \hat{v}_{n}(x) \rightarrow \hat{v}(x)$. Then $\hat{v}_{n}(x) \rightarrow \hat{v}(x)$ uniformly on every compact $\sigma \subset \boldsymbol{R}^{m}$.

Suppose that we given the sequence $b_{x}^{n}$ of function of class $\operatorname{CP}\left(\boldsymbol{R}^{m}\right)$ with canonical representations

$$
b_{x}^{n}=i\left\langle a_{n}, x\right\rangle-\frac{1}{2}\left\langle B_{n} x, x\right\rangle+\int_{\boldsymbol{R}^{m}}\left(e^{i\langle x, y\rangle}-1<\frac{i\langle x, y\rangle}{1+|y|^{2}}\right) \frac{1+|y|^{2}}{|y|^{2}} d \mu_{n}(y) .
$$

LEMMA 8.1. Suppose that for every $x b_{x}^{(n)} \rightarrow 0$. Then $a_{n} \rightarrow 0, B_{n} x \rightarrow 0$ for every $x \in \boldsymbol{R}^{m}$ uniformly on every compact and $\mu_{n}\left(\boldsymbol{R}^{m}\right) \rightarrow 0$.

Proof. By the Levy-Khintehine theorem

$$
e^{b_{x}^{(n)}}=\hat{v}_{n}(x) \quad\left(x \in \boldsymbol{R}^{m}\right)
$$

for infinitely divisible distribution $v_{n}(\cdot)$ on $B\left(\boldsymbol{R}^{m}\right)$. By our assumption $\hat{v}_{n}(x)$ converges to $\hat{v}(x) \equiv 1$. Consequently, since $\hat{v}(\cdot)$ is a characteristic function of the degenerated distribution concentrated at $x=0$, we infer by (L) that $\hat{\nu}(x) \rightarrow 1$ uniformly on every compact. It follows that $\left|\hat{v}_{n}(x)\right|$ $=e^{\operatorname{Re} b_{x}^{n} \rightarrow 1}$ uniformly on every compact. Hence $\operatorname{Re} b_{x}^{n} \rightarrow 0$ uniformly on every compact. Hence

$$
-\operatorname{Re} b_{x}^{n}=\frac{1}{2}\left\langle B_{n} x, x\right\rangle+\int_{n^{m}}(1-\cos \langle x, y\rangle) \frac{1+|y|^{2}}{|y|^{2}} d \mu_{n}(y) \rightarrow 0
$$

uniformly on every compact. But $\mu_{n} \geqslant 0$ and $1-\cos \langle x, y\rangle \geqslant 0$. It follows that $B_{n} x \rightarrow 0$ on every compact uniformly, and

$$
\int_{\mathbf{R}^{m}}(1-\cos \langle x, y\rangle) \frac{1+|y|^{2}}{|y|^{2}} d \mu_{n}(y) \rightarrow 0
$$

uniformly in $x$ on every compact.

If $K_{h}=\left\{x:\left|x_{i}\right| \leqslant h, x=\left(x_{1}, \ldots, x_{n}\right), i=1, \ldots, n\right\}$, where $h>0$ wo conclude that

$$
\begin{aligned}
c_{n} & =\int_{\boldsymbol{R}^{m}} \int_{K_{h}}(1-\cos \langle x, y\rangle) \frac{1+|y|^{2}}{|y|^{2}} d \mu_{n}(y) d x \\
& =(2 h)^{m} \int_{\boldsymbol{R}^{m}}\left(1-\prod_{l_{k \mid 1}}^{m} \frac{\sin h y_{k}}{h y_{k}}\right) \frac{1+|y|^{2}}{|y|^{2}} d \mu_{u}(y) \rightarrow 0 .
\end{aligned}
$$


By previous lemma, $\frac{c_{n}}{(2 h)^{m} \eta_{m}(h)} \geqslant \mu_{n}\left(\boldsymbol{R}^{m}\right) \geqslant 0$ and consequently $\mu_{n}\left(\boldsymbol{R}^{m}\right)$

$\rightarrow 0$. On the other hand, by (6.3)

$$
z(x, y)=\left|\left(e^{i\langle x, y\rangle}-1-\frac{i\langle x, y\rangle}{|y|^{2}}\right) \frac{1+|y|^{2}}{|y|^{2}}\right| \leqslant 2+\frac{|x|^{2}}{2}
$$

for $x \in \boldsymbol{R}^{m}, y \neq 0$. Since $\mu_{n}(\{0\})=0$, we get that

$$
0 \leqslant \int_{\boldsymbol{R}^{m}} z(x, y) d \mu_{n}(y) \leqslant\left(2+\frac{|x|^{2}}{2}\right) \mu_{n}\left(\boldsymbol{R}^{m}\right) \rightarrow 0
$$

which, since $B_{n} x \rightarrow 0$ uniformly on every compact, implies that $\left\langle a_{n}, x\right\rangle$ $\rightarrow 0$ uniformly on every compact. This plainly proves that $a_{n} \rightarrow 0$ which completes the proof of our lemma.

We define as before for $x \in \boldsymbol{R}^{m}$

$$
\begin{aligned}
& h(x, y)=\left(e^{i\langle x, y\rangle}-1-\frac{i\langle x, y\rangle}{1+|y|^{2}}\right) \frac{1+|y|^{2}}{|y|^{2}} \quad \text { for } y \neq 0, \\
& h(x, 0)=0 .
\end{aligned}
$$

Suppose that $\mu_{n}, \mu$ are positive measures on $B\left(\boldsymbol{R}^{m}\right)$ and

$$
\mu_{n}(\sigma) \rightarrow \mu(\sigma) \quad \text { for } \sigma \in B\left(\boldsymbol{R}^{m}\right)
$$

and $\mu_{n}(\{0\})=0, n=1,2, \ldots$ Then, of course, $\mu(\{0\})=0$ and, moreover,

$$
\int_{\boldsymbol{R}^{m}} h(x, y) d \mu_{n}(y) \rightarrow \int_{\boldsymbol{R}^{m}} h(x, y) d \mu(y)
$$

uniformly in $x$ on every compact $\sigma \subset \boldsymbol{R}^{m}$.

Proof. For $\eta>0$ we write $S_{\eta}=\left\{x \in \boldsymbol{R}^{m}:|x| \leqslant \eta\right\}$. Since $|h(x, y)|$ $\leqslant 2+\eta^{2} / 2=\eta_{1}$ for $x \in S_{\eta}$ and $y$ arbitrary, it is sufficient to prove (8.5) for $\sigma=S_{\eta}$. For $\varepsilon>0$ there is $\eta_{2}>0$ such that $\mu\left(\boldsymbol{R}^{m}-S_{\eta_{2}}\right)<\varepsilon / 6 \eta_{1}$ which implies that for $n \geqslant n\left(\eta_{2}\right)$

$$
\left|\int_{\boldsymbol{R}^{m}} \int_{-S_{\eta_{2}}} h(x, y) d\left(\mu_{n}-\mu\right)(y)\right| \leqslant \varepsilon / 3, \quad x \in S_{\eta} .
$$

If $\eta_{3}>0$ is small enough, then for $n \geqslant n\left(\varepsilon, \eta_{3}\right)$

$$
\mu_{n}\left(S_{\eta_{3}}\right)+\mu\left(S_{\eta_{3}}\right) \leqslant \varepsilon / 3 \eta_{1},
$$

because $\mu(\{0\})=0$. It follows that

$$
\left|\int_{S_{\eta_{3}}} h(x, y) d\left(\mu_{n}-\mu\right)\right| \leqslant \varepsilon / 3 \quad \text { for } n \geqslant n\left(\varepsilon, \eta_{3}\right), \quad x \in S_{\eta} .
$$

We take $\eta_{3}$ so small that $S_{\eta_{3}} \subset S_{\eta_{2}}$. We define

$$
r=\max \left(\mu\left(S_{\eta_{2}}-S_{\eta_{3}}\right), \sup _{n} \mu_{n}\left(S_{\eta_{2}}-S_{\eta_{3}}\right)\right) \text {. }
$$


Since $h(x, y)$ is uniformly continuous on $S_{\eta} \times\left(S_{\eta_{2}}-S_{\eta_{3}}\right)$, for $\varepsilon>0$ there is a measurable partition $\sigma_{1}, \ldots, \sigma_{p}$ of $S_{\eta_{2}}-S_{\eta_{3}}$ and points $y_{i} \in \sigma_{i}(i=1, \ldots$ $\ldots, p)$ such that

$$
\left|h\left(x, y_{i}\right)-h(x, y)\right| \leqslant \frac{\varepsilon}{12(r+1)}, \quad x \in S_{\eta} \quad \text { for } y^{\prime} \in \sigma_{i} .
$$

Hence for $y \in S_{\eta_{2}}-S_{\eta_{3}}, x \in S_{\eta}$

$$
\left|\sum_{j \mid 1}^{p} h\left(x, y_{j}\right) \chi_{\sigma_{i}}(y)-h(x, y)\right| \leqslant \varepsilon / 12(r+1) .
$$

Since $\mu_{n}\left(\sigma_{j}\right) \rightarrow \mu\left(\sigma_{j}\right)$,

$$
\left|\sum_{j \mid 1}^{p} h\left(x, y_{j}\right) \mu_{n}\left(\sigma_{j}\right)-\sum_{j \mid 1}^{p} h\left(x, y_{j}\right) \mu\left(\sigma_{j}\right)\right| \leqslant \varepsilon / 12(r+1)
$$

for $n \geqslant n\left(\sigma_{1}, \ldots, \sigma_{p}\right), x \in S_{\eta}$. But the previous inequality proves that

$$
\begin{aligned}
& \left|\sum_{j \mid 1}^{p} h\left(x, y_{j}\right) \mu_{n}\left(\sigma_{j}\right)-\int_{s_{\eta_{2}}-S_{\eta_{3}}} h(x, y) d \mu_{n}(y)\right| \leqslant \frac{\varepsilon r}{12(r+1)} \leqslant \frac{\varepsilon}{12} \\
& \left|\sum_{j \mid 1}^{p} h\left(x, y_{j}\right) \mu\left(\sigma_{j}\right)-\int_{s_{\eta_{2}}-s_{\eta_{3}}} h(x, y) d \mu(y)\right| \leqslant \frac{\varepsilon r}{12(r+1)} \leqslant \frac{\varepsilon}{12}
\end{aligned}
$$

and consequently for $x \in S_{\eta}$

$$
\left|\int_{s_{\eta_{2}}-s_{\eta_{3}}} h(x, y) d\left(\mu_{n}-\mu\right)\right| \leqslant \varepsilon / 4
$$

for $n \geqslant n\left(\sigma_{1}, \ldots, \sigma_{p}\right)$. Summing up,

$$
\left|\int_{\boldsymbol{R}^{m}} h(x, y) d\left(\mu_{n}-\mu\right)\right| \leqslant \varepsilon \quad \text { for } x \in S_{\eta}
$$

and $n \geqslant \max \left(n\left(\eta_{2}\right), n\left(\varepsilon, \eta_{9}\right), n\left(\sigma_{1}, \ldots, \sigma_{p}\right)\right)$.

If $b_{x}(f, g)$ is in $\mathrm{CP}\left(\boldsymbol{R}^{m} ; \mathscr{E}\right)$, then

$$
b_{x}(f, g)=u_{f, g}(x)-\frac{1}{2} G(f, g ; x, x)+\int_{\boldsymbol{R}^{m}} h(x, y) d\left(E_{y} R f, R g\right),
$$

where $(E(\sigma) R f, R g)=\mu_{f, g}(\sigma)$ is the corresponding canonical representation of $\mu_{f, g}, R: \mathscr{E} \rightarrow K=$ the minimal Hilbert space with inner product $(\cdot, \cdot)$. Representation $(8.6)$ will be called the spectral representation of $b_{x}(f, g)$.

Proposition 8.0. If $b_{x}(f, g)$ of class $\mathrm{CP}\left(\boldsymbol{R}^{m}, \mathscr{E}\right)$ has the spectral representation (8.6) and $f_{n} \in \mathscr{E}$ and $b_{x}\left(f_{n}, f_{n}\right) \rightarrow 0$ for $x \in \boldsymbol{R}^{m}$, then $R f_{n} \rightarrow 0$. 
Proof. We put $b_{x}^{(n)}=b_{x}\left(f_{n}, f_{n}\right)$ and, using notations of Lemma 8.1 conclude that $\left(R f_{n}, R f_{n}\right)=\left(E\left(\boldsymbol{R}^{m}\right) R f_{n}, R f_{n}\right)=\mu_{n}\left(\boldsymbol{R}^{m}\right) \rightarrow 0$ which proves the claim.

It is sufficient for analytical purposes to deal with metric linear spaces.

We are able to prove now the following theorem:

THEOREM 8.0. Let $b_{x}(f, g)$ of class $\mathrm{CP}\left(\boldsymbol{R}^{m} ; \mathscr{E}\right)$ have the spectral representation (8.6). If $\mathscr{E}$ is a metric space and the function $f \rightarrow b_{x}(f, f)$ is continuous at $f=0$ for every $x$, then the operator $R$ is continuous.

Proof. Let $f_{n} \rightarrow 0$ in $\mathscr{E}$. Then $b_{x}\left(f_{n}, f_{n}\right) \rightarrow 0$ for every $x$. By Proposition 8.0 $R f_{n} \rightarrow 0$.

Our basic result is the following:

THEOREM 8.1. Let $b_{x}(f, g)$ of class $\mathrm{CP}\left(\boldsymbol{R}^{m} ; \mathscr{E}\right)$ have the spectral rep. resentation (8.6). Let $\mathscr{E}$ be a metric space. Suppose that $b_{x}(f, g)$ is jointly continuous in $f, g$ for every $x \in \boldsymbol{R}^{m}$, i.e. by polarization formula $b_{x}(f, f)$ is continuous in $f$ for $x \in \boldsymbol{R}^{m}$. Suppose that $f_{n} \rightarrow f, g_{n} \rightarrow g$. Then the following holds true:

(i) $R$ is a continuous operator;

(ii) $u_{f_{n}, g_{n}}(x) \rightarrow u_{f, g}(x)$ uniformly on every compact $\cup \subset \boldsymbol{R}^{m}$;

(iii) $G\left(f_{n}, g_{n} ; x, y\right) \rightarrow G(f, g$; $x, y)$ uniformly in $(x, y)$ on every compact $a \subset \boldsymbol{R}^{m} \times \boldsymbol{R}^{m}$; $\boldsymbol{\sigma} \subset \boldsymbol{R}^{m}$.

(iv) $\int_{\boldsymbol{R}^{m}} h(x, y) d \mu_{f_{n}, g_{n}} \rightarrow \int_{\boldsymbol{R}^{m}} h(x, y) d \mu_{f, g}$ uniformly in $x$ on every compact

Proof. (i) follows from Theorem 8.0. Since the parts of the spectral representation of $b_{x}(f, g)$ are bilinear in $f, g$, it is sufficient to prove our assertion for $f_{n}=g_{n}, f=g$. We define $\mu_{n}(\sigma)=\left(E(\sigma) R f_{n}, R f_{n}\right), \mu(\sigma)$ $=(E(\sigma) R f, R f)$. It follows that $\mu_{n}(\{0\})=\mu(\{0\})=0$. The continuity of $R$ yields that (8.4) holds true. Consequently, by (8.5) (iv) holds true. Now, $b_{x}\left(f_{n}, f_{n}\right) \rightarrow b_{x}(f, f)$ by assumption, for $x \in \boldsymbol{R}^{m}$. Hence

$$
\hat{\nu}_{n}(x)=e^{b_{x}\left(f_{n}, f_{n}\right)} \rightarrow \hat{\nu}(x)=e^{b_{x}(f, f)}
$$

for every $x \in \boldsymbol{R}^{m}$, in fact uniformly in $x$ on every compact $\sigma \subset \boldsymbol{R}^{m}$ by (L). It follows that

$$
e^{b_{x}\left(f_{n}, f_{n}\right)-b_{x}(f, f)} \rightarrow 1
$$

uniformly on $\sigma$, which just like in the proof of (8.5) implies that

$$
b_{x}\left(f_{n}, f_{n}\right) \rightarrow b_{x}(f, f)
$$

uniformly on $\sigma$. Since (iv) holds true, we conclude that

$$
u_{f_{n}, f_{n}}(x)-\frac{1}{2} G\left(f_{n}, f_{n} ; x, x\right) \rightarrow u_{f, f}(s)-\frac{1}{2} G(f, f ; x, x)
$$


uniformly on $\sigma$. Since $u_{f_{n}, f_{n}}(x), u_{f, f}(x)$ are purely imaginary and $G\left(f_{n}, f_{n}\right.$; $x, x), G(f, f ; x, x)$ are real, we infer therefore that $u_{f_{n}, f_{n}}(x) \rightarrow u_{f, f}(x)$ uniformly on $x$ which proves that (ii) is true, as well that $G\left(f_{n}, f_{n} ; x, x\right)$ $\rightarrow G(f, f ; x, x)$ uniformly in $x$ on $\sigma$. Since $G(f, g ; x, y)$ is real bilinear in $x, y$ (iii) follows from the polarization formula for $G$ with respect to $x, y$. This completes the proof.

The short reformulation of the above theorem reads as follows:

(8.7) If $\mathscr{E}$ is a metric space and $b_{x}(f, g)$ of class $\mathrm{CP}\left(\boldsymbol{R}^{m} ; \mathscr{E}\right)$ is jointly continuous in $f, g$ for every $x \in \boldsymbol{R}^{m}$, then the parts of the canonical representation of $b_{x}(f, g)$ are jointly continuous in $f, g$ uniformly for $x$ varying over an arbitrary compact in $\boldsymbol{R}^{m}$.

The following definition is now in order:

Definition 8.0. Let $\mathscr{E}$ be a complex linear m etric space. We denote by $\bar{L}(\mathscr{E})$ the totality of linear maps $A$ from $\mathscr{E}$ into the space $\mathscr{E}^{*}$ of antilinear continuous functionals on $\mathscr{E}$, such that $(A f)(g)$ is jointly continuous in $f, g$.

It is easy to see that there is one-to-one correspondence between jointly continuous bilinear forms $l(f, g)$ and operators in $\bar{L}(\mathscr{E})$, where $l(\cdot, \cdot)$ corresponds to $A_{l}$ if and only if $\left(A_{l} f\right)(g)=l(f, g)$ for $f, g \in \mathscr{E}$.

Let us say that the function $x \rightarrow B(x) \in \bar{L}(\mathscr{E})\left(x \in \boldsymbol{R}^{m}\right)$ is of class $\overline{\mathbf{C P}}\left(\boldsymbol{R}^{m} ; \mathscr{E}\right)$, if the function $b_{x}(f, g)=(B(x) f)(g)$ is of class $\operatorname{CP}\left(\boldsymbol{R}^{m} ; \mathscr{E}\right)$. The operator version of Theorem 8.1 reads as follows:

THeOREM 8.2. Let $\boldsymbol{B}(x)$ be a function of class $\overline{\mathbf{C P}}\left(\boldsymbol{R}^{m} ; \mathscr{E}\right)$, where $\mathscr{E}$ is a complex linear metric space. Then there are the unique functions $i A(x)$, $\boldsymbol{G}(x, y)$ of class $\overline{\mathbf{C P}}\left(\boldsymbol{R}^{m} ; \mathscr{E}\right)$ and the unique function $\boldsymbol{F}(\cdot): B\left(\boldsymbol{R}^{m}\right) \rightarrow \bar{L}(\mathscr{E})$ such that $(F(\sigma) f)(g)$ is a complex measure on $B\left(\boldsymbol{R}^{m}\right)$ for $f, g \in \mathscr{E}$ satisfying the following conditions:

(i) $(A(x) f)(f)$ is real for $x \in \boldsymbol{R}^{m}, f \in \mathscr{E}$, real linear in $x$ and $\left(A(x) f_{n}\right)\left(g_{n}\right)$ $\rightarrow(A(x) f)\left(g_{n}\right)$ uniformly for $x \in \sigma=$ compact $\subset \boldsymbol{R}^{m}$ if $f_{n} \rightarrow f, g_{n}$ $\rightarrow g ;(A(x) f)(g)$ is continuous in $x$.

(ii) $G(x, y)$ is real bilinear in $x, y,(G(x, x) f)(f) \geqslant 0$ for $x \in \boldsymbol{R}^{m}, f \in \mathscr{E}$ and $(G(x, y) f)(g)$ is continuous in $x, y$ and in $f, g$ uniformly for $(x, y)$ varying over any compact in $\boldsymbol{R}^{m} \times \boldsymbol{R}^{m}$;

(iii) $(F(\sigma) f)(f) \geqslant 0$ for $\sigma \in B\left(\boldsymbol{R}^{m}\right), f \in \mathscr{E}$ and the formula

$$
B(x)=i A(x)-\frac{1}{2} G(x, x)+\int_{\mathbf{R}^{m}} h(x, y) d F_{y}, \quad x \in \boldsymbol{R}^{m}
$$

holds true, where the last term is the unique operator in $\bar{L}(\mathscr{E})$ such that $\int_{\boldsymbol{R}^{m}} h(x, y) d\left(F_{y} f\right)(g)(f, g \in \mathscr{E})$ exists and equals to the Poisson part of $(B(x) f)(g)$. 
Proof. The uniqueness properties follow from results of Section 6 . $i A(x), G(x, y)$ are defined by equalities $i(A(x) f)(g)=u_{f, \theta}(x),(G(x, y) f)(g)$ $=G(f, g ; x, y)$ and $(F(\sigma) f)(g)=(E(\sigma) R f, R g)$ of spectral representation (8.6). Then the assertion follows from Theorem 8.1.

CoRollary 8.0. If $\mathscr{E}$ is an F-space (see [4], [30]), i.e. linear metric complete space with translation invariant metric or if $\mathscr{E}$ is metric barrelled locally convex (J. Horvath [10]), then if $b_{x}(f, g)$ is separately continuous in $f$ and in $g$ for every $x \in \boldsymbol{R}^{m}$ then Theorem 8.1 as well Theorem 8.2 apply to $b_{x}(f, g)=(B(x) f)(g)$. Consequently, if $\mathscr{E}$ is a normed space or especially a complex Hilbert space, then the separate continuity in $f, g$ for $x \in \boldsymbol{R}^{m}$ of $b_{x}(f, g)$ imply that $b_{x}(f, g)=(B(x) f)(g)$ with $B(\cdot)$ in class $\overline{\mathbf{C P}}\left(\boldsymbol{R}^{m} ; \mathscr{E}\right)$. This is true if $\mathscr{E}$ is a Fréchet space, i.e. a locally convex, metvizable complete linear space with metries

$$
\varrho(f, g)=\sum_{n \mid 1}^{\infty} \frac{1}{2^{n}} \frac{p_{n}(f-g)}{1+p_{n}(f-g)}
$$

where $\left\{p_{n}\right\}$ is a sequence of seminorms $p_{n}$ which separate in $\mathscr{E}$, i.e. if $p_{n}(f)=0$ for all $n$ then $f=0$.

The compact and nice formulation of Theorem 8.2 appears when $\mathscr{E}$ is a complex Hilbert space with the inner product $(\cdot, \cdot)$. In what follows we refer to [2], [21] for semi spectral integrals.

THEOREM 8.3. If $\mathscr{E}$ is a complex Hilbert space and $B(x) \in L(\mathscr{E})$ is of class $\overline{\mathrm{CP}}\left(\boldsymbol{R}^{m} ; \mathscr{E}\right)$, then there are unique functions $A(x), G(x, y) \in L(\mathscr{E})$ for $x, y \in \boldsymbol{R}^{m}$ and the unique semi-spectral measure $F(\sigma) \in L(\mathscr{E})$ on $B\left(\boldsymbol{R}^{m}\right)$ such that

$$
\boldsymbol{B}(x)=i \boldsymbol{A}(x)-\frac{1}{2} G(x, x)+\int_{\boldsymbol{R}^{m}} h(x, y) d \boldsymbol{F}_{y}, \quad x \in \boldsymbol{R}^{m},
$$

where $h(x, 0)=0, h(x, y)=\left(e^{i\langle x, y\rangle}-1-\frac{i\langle x, y\rangle}{1+|y|^{2}}\right) \frac{1+|y|^{2}}{|y|^{2}}$ for $y \neq 0$ and

(i) $A(x)=A(x)^{*}$ is real linear in $x$;

(ii) $G(x ; x) \geqslant 0$ for $x \in \boldsymbol{R}^{m}$ and $G(x, y)$ is real bilinear in $x, y$;

(iii) $A(x)$ and $G(x, y)$ are continuous in $x,(x, y)$ resp. in the operator norm. (iv) The Poisson part $\int_{\boldsymbol{R}^{m}} h(x, y) d F_{y}$ is strongly continuous in $x, F(\{0\})$
$=0$,

and consequently $B(x)$ is strongly continuous in $x$ and continuous in operator norm if and only if the Poisson part shares this property (for instance, when the closed support of $F, S_{F}$ is compact). 
Proof. It follows from the previous theorem that only the continuity properties need the proof. Let $e_{1}, \ldots, e_{m}$ be the orthonormal basis in $\boldsymbol{R}_{m}^{m}$. Then for $x=\sum_{j \mid 1}^{m} a_{j} e_{j}, y=\sum_{j ! 1}^{m} \beta_{j} e_{j}$ which implies that $A(x)$ $=\sum_{j \mid 1}^{m} \beta_{j} A\left(e_{j}\right)$ and $G(x, y)=\sum_{j, k \mid 1}^{m} G\left(e_{j}, e_{k}\right) \alpha_{j} \beta_{k}$ which proves (iii).

The proof of (iv) runs as follows: by Proposition 2.0 (in fact, by the classical Naimark's theorem - see [22], [24]) for $F$ of (8.8) we have $(F(\sigma) f, g)=(E(\sigma) R f, R g)_{K}$ with some minimal space $K=\underset{\sigma \in B\left(R^{m}\right)}{\bigvee} E(\sigma) R \mathscr{E}$, spectral measure $E(\cdot)$ in $K$ and a continuous operator $R: \mathscr{E} \rightarrow K$. It follows that $E(\{0\})=0$. Let $h\left(x_{n}, y\right)-h\left(x_{0}, y\right)=p_{n}(y)$ for $x_{n} \rightarrow x_{0}$. The inequality (6.3) implies that $p_{n}(y)$ is bounded sequence of functions and $p_{n}(y) \rightarrow 0$ a.e. with respect to $E$. It follows that for $f \in \mathscr{E}$

$$
\left\|\int_{\boldsymbol{R}^{m}} p_{n}(y) d F_{y} f\right\|^{2} \leqslant\left\|R^{*}\right\|^{2} \int_{\boldsymbol{R}^{m}}\left|p_{n}(y)\right|^{2} d\left(E_{y} R f, R f\right)_{K} \rightarrow 0
$$

by dominated convergence.

CoRoLlary 8.1. This is a simple exercise to formulate the analogon of Theorem 8.3 for $\mathscr{E}$ being a Banach space.

CoRollaky 8.2. If $\boldsymbol{R}^{m}=\boldsymbol{R}^{1}$, then (8.9) takes the form

$$
B(x)=i A x-\frac{1}{2} G \cdot x^{2}+\int_{-\infty}^{+\infty}\left(e^{i x y}-1-\frac{i x y}{1+y^{2}}\right) \frac{1+y^{2}}{y^{2}} d E_{y}, \quad F(\{0\})=0,
$$

where $A^{*}=A=A(1), G=G(1,1) \geqslant 0$ according to notation of Theorem 8.2. Defining

we get formally

$$
\tilde{h}(x, y)= \begin{cases}\left(e^{i x y}-1-\frac{i x y}{1+y^{2}}\right) \frac{1+y^{2}}{y^{2}} & \text { for } y \neq 0, \\ -\frac{1}{2} x^{2} & \text { for } y=0,\end{cases}
$$

$$
B(x)=i A x+\int_{-\infty}^{+\infty} h(x, y) d \tilde{F}_{y}
$$

where $\tilde{F}(\sigma)=F(\sigma)$ if $0 \notin \sigma, \tilde{F}(\{0\})=G(1,1)$.

If $\mathscr{E}$ is a complex Hilbert space then according to Theorem 8.3 we have the unique representation

$$
B(x)=i A x+\int_{-\infty}^{+\infty} \tilde{h}(x, y) d \tilde{H}_{y},
$$

$A=A^{*}, \tilde{F}-a$ semi-spectral measure. By Theorem 6.3 the last formula gives the general form of $B(\cdot)$ in class $\overline{\mathbf{C P}}\left(\boldsymbol{R}^{1} ; \mathscr{E}\right)$, and $B(\cdot)$ is conditionally positive definite, i.e. $b_{x}(f, g)$ is. 
CoRollary 8.3. Following notation of Section 7, if $\mathscr{E}$ is a complex Hilbert space, all formula as the Yaglom one, the formula for the structural functions, etc. can be written in an operator version for $B(\cdot)$ of class $\overline{\mathbf{C P}}\left(\boldsymbol{R}^{m} ; \mathscr{E}\right)-$ for instance, there corresponds to the structural function related to $B(\cdot)$ of Poisson type of Theorem 8.3 the semi-spectral integral

$$
\int_{\mathbf{R}^{m}}\left(e^{i\langle x, s\rangle}-e^{i\langle y, s\rangle}\right) \overline{\left(e^{i\left\langle x^{\prime}, s\right\rangle}-e^{i\left\langle y^{\prime}, s\right\rangle}\right)} \frac{1+|y|^{2}}{|y|^{2}} d F_{y}
$$

and to $Y_{0}(x)$ the formula

$$
Y_{0}(x)=\int_{\boldsymbol{R}^{m}}\left(e^{i\langle x, s\rangle}-1\right) \eta(s) d F_{s}
$$

where $F$ is the semi-spectral measure appearing in (8.9) when $A(x)=G(x, x)$ $=0$, because $\boldsymbol{B}(\cdot)$ is of Poisson type. If $\boldsymbol{R}^{m}=\boldsymbol{R}^{1}$, then we get therefore for $F=\tilde{F}$ of (8.11) the generalizations of Masani's [18] results for operator valued functions with $\eta(s)=(i+s) / s$.

\section{References}

[1] N. Aronszajn, Theory of reproducing kernels, Trans. Amer. Math. Soc. 68 (1950), p. 337-404.

[2] S. K. B erberian, Notes on spectral theory, Van Nostr. Math. Studies 5, Princeton 1966.

[3] R. Cuppens, Decomposition of multivariate probability, Prob. Math. Statist. Acad. Press, New York 1975.

[4] N. Dunford and J. T. Schwartz, Linear operators, Part I, Intersc. Publ., New York 1958.

[5] R. K. Getoor, The shift operator for non-stationary processes, Duke Math. J. 23 (1956), p. 175-187.

[6] I. I. Gichman and A. V. Skorohod, The theory of stochastic processes, Vol. I, Springer Verlag, Berlin 1974.

[7] J. Górniak, Remarks on positive definite operator valued functions in linear spaces, in Probability Theory on Vector Spaces, Springer Lect. Notes 656 (1978), p. 37-44.

[8] A. Guichardet, Symmetric Hilbert spaces and related topics, Springer Lect. Notes 261 (1972).

[9] P. Halmos, Measure theory, D. Van Nostrand, New York 1950.

[10] J. Horváth, Topological vector spaces and distributions, Vol. I, London 1966.

[11] A. Kolmogorov, Stationary sequences in Hilbert space, Bull. Math. Univ. Moscov 2 (1941), p. 1-49.

[12] A. Koranyi und B.Sz.-Nagy, Operatortheoretische Behandlung und Verallgemeinerung eines Problem Kreises in der Komplexen Funktionen Theorie, Acta Math. 100 (1958), p. 171-202.

[13] M. G. Krein, On the logarithm of an infinitely decomposable Hermile-positive functions, Dokl. Akad. Nauk SSSR 45 (1944), p. 91-94.

[14] -, Hermitian positive kernels on homogeneous spaces, I, Ukr. Mat. I. (1948); Amer. Math. Soc. Transl. Ser. 2, vol. 34, Providence R. I., 1963. 
[15] R. A. K unze, Positive definite operator valued kernels and unitary representations, Proc. Conf. UC, Irvine, ed. B. R. Geldbaum, Acad. Press, London 1967, p. 235-247.

[16] R. G. Laha and V. K. Rohatgi, Probability theory, Wiley Ser. Prob. Math. Statist., New York 1979.

[17] Yu. V. Linnik and I. V. Ostrovskii, Decomposition of random variables and random vectors, Amer. Math. Soc., Providence R. I., 1977.

[18] P. Masani, Helixes in Hilbert space, Teor. Ver. Prim. (SSSR) 17 (1972), p. 3-20; transl. SIAM 17, p. 1-19.

[19] -, On infinitely decomposable probability distributions and helical varieties in Hilbert space, J. Multiv. Anal. 3 (1973), p. 209-223.

[20] -, An explicit treatment of dilation theory, preprint Aut. 1975.

[21] W. Mlak, Unitary dilations of contraction operators, Dissert. Math. 46, Warszawa 1965 , p. 1-91.

[22] -, Dilations of Hilbert space operators (general theory), ibidem 153, Warszawa 1978 , p. $1-65$.

[23] -, A note on general dilation theorems, in Spectral theory, Banach Center Publ., vol. 8, PWN, Warsaw 1982.

[24] M. A. Naimark, On a representation of additive operator set functions, Dokl. A.N. USSR 41 (1943), p. 359-361.

[25] J. von Neumann and I. J. Schönberg, Fourier integrals and metric geometry, Trans. Amer. Math. Soc. 50 (1941), p. 226-251.

[26] K. R. Parthasarathy, Probability measures on metric spaces, Acad. Press, New York 1967.

[27] -, and K. Schmidt, Positive definite kernels, continuous tensor products and central limit theorems of probability theory, Springer Lect. Notes 272 (1972).

[28] G. B. Pedrick, Theory of reproducing kernels in Hilbert spaces of vector valued functions, Univ. of Kansas, Techn. Rep. 19, Lawrence 1957.

[29] M. Reed and B. Simon, Methods of modern mathematical physics, I. (1972), II (1975), New York.

[30] W. Rudin, Functional analysis, McCraw-Hill Comp., New York 1973.

[31] -, Real and complex analysis, New York 1966.

[32] I. J. Schönberg, Metric spaces and positive definite functions, Trans. Amer. Math. Soc. 44 (1938), p. 522-536.

[33] B. Sz.-Nagy, Extensions of linear transformation in Hilbert space which extend beyond this space, Appendix to F. Riesz and B. Sz.-Nagy, Leçons d'anal. fonct., Fred. Ungar, New York 1960.

[34] S. R. S. Varadhan, Limit theorems for sums of independent random variables with values in a Hilbert space, Sankhy a Ser. A, 24 (1962), p. 213-238.

[35] A. M. Yaglom, Some classes of random fields in n-dimensional space related to. stationary random processes, Th. Prob. Appl. (USSR) (1957), transl. SIAM 4, p. 289-320.

[36] -, Corelation theory of processes with stationary n-increments, Math. Sb. 37 (1955), p. 141-196.

INSTITUTE OF MATHEMATICS, POLISH ACADEMY OF SCIENCES

31-027 IKRAKOW, UL. SOLSKIEGO 30, POLAND

Resu par la Rédaction le 1.10.1980 